\title{
Studies of Nerve-Muscle Interactions in Xenopus Cell Culture: Analysis of Early Synaptic Currents
}

\author{
Jane Evers, ${ }^{a}$ Mark Laser, Yi-an Sun, ${ }^{b}$ Zuo-ping Xie, ${ }^{c}$ and Mu-ming Poo \\ Section of Molecular Neurobiology, Yale University School of Medicine, New Haven, Connecticut 06510
}

We have studied the spontaneous and nerve-evoked synaptic currents during the initial period of nerve-muscle contact in Xenopus cell cultures. The precise timing of the contact was achieved by physically manipulating embryonic muscle cells into contact with co-cultured spinal neurons. Previous studies have shown that physical contact of the muscle membrane induces pulsatile release of acetylcholine (ACh) from the growth cone of these neurons, resulting in spontaneous synaptic currents (SSCs) in the muscle cell within seconds following the contact. In the present work, we first showed that these SSCs at the manipulated nervemuscle contacts are similar to those observed at naturally occurring synapses. We then examined the possible cellular mechanisms responsible for the marked variation in SSC amplitude and showed that it most likely results from differences in either the amount of ACh contained in each release event or the extent of close membrane apposition near the release sites.

During the first $20 \mathrm{~min}$ following the nerve-muscle contact, there was an increase in the frequency and mean amplitude of the SSCs. During a similar period, the evoked synaptic currents (ESCs), which were induced by suprathreshold electrical stimulation of the neuronal soma, also showed an increase in the mean amplitude and a reduction in the delay of onset following the stimulus. These postcontact changes in the efficacy of synaptic transmission may be related to an increase in the total area of close membrane apposition between the nerve and muscle cells. This was suggested by the finding that neurite-muscle adhesion increases over a similar postcontact period. The transition from low- to highefficacy transmission during the early phase of contact may reflect the process of selective adhesion between the cells, and thus signify the formation of specific synapse.

Analysis of the fluctuation in the ESC amplitude at the early nerve-muscle contact suggests that evoked release of ACh occurs as multiples of a quantal unit. However, this unit is apparently related to only a small subpopulation of SSCs of relatively high amplitudes. Depleting extracellular $\mathrm{CA}^{2+}$ totally abolished the ESCs but left many of the SSCs

Received Apr. 14, 1988; revised Sept. 20, 1988; accepted Sept. 26, 1988

We thank Dan Sanes for helpful discussions. This work was supported by National Institutes of Health (NS 22764, NS 12961) and National Science Foundation (BNS 8545366).

Correspondence should be addressed to Dr. Mu-ming Poo, Department of Biological Sciences, Fairchild Center, Columbia University, New York, NY 10027.

a Present address: Department of Anatomy, University College, London.

b Present address: Department of Biology, Yale University, New Haven, CT.

' Present address: Department of Biology, Tsinghua University, Beijing, China. Copyright (C 1989 Society for Neuroscience $0270-6474 / 89 / 051523-17 \$ 02.00 / 0$ unaffected. These findings indicate that the cellular mechanism responsible for regulating evoked $A C h$ release is substantially different from that of spontaneous $\mathrm{ACh}$ release during the early period of synaptogenesis.

Early nerve-muscle interactions are important for the development of the neuromuscular synapse. These interactions are responsible for the initial nerve-muscle recognition, the development of selective adhesion, the modification of the growth cone to a transmitting nerve terminal, and the localization of specific synaptic components. While little is known about the molecular and cellular mechanisms underlying these events, they are likely to begin within minutes following the nerve-muscle contact. For studying events of this time scale, the control of the timing of contact becomes critical. It was shown recently that in Xenopus cell culture the timing of synaptic contact could be controlled by physically manipulating the position of the muscle cell relative to that of the neurite (Chow and Poo, 1985; Xie and Poo, 1986). This approach has led to the finding that one of the first events of nerve-muscle interaction is a contactdependent inducation of spontaneous, pulsatile release of acetylcholine $(\mathrm{ACh})$ from the embryonic spinal neuron. The release occurs within seconds following the contact and results in pulsatile synaptic currents of varying amplitudes in the muscle cell (Xie and Poo, 1986). Functional synaptic transmission, as indicated by the synaptic current evoked by a nerve impulse was also found to occur within the first few minutes of contact (Kidokoro and Yeh, 1982; Sun and Poo, 1987). The present report describes further analysis of both the spontaneous and the evoked synaptic currents during the early phase of nervemuscle contact. We have compared these currents at manipulated contacts with those occurring at natural nerve-muscle contacts in the same culture. The origin of the amplitude fluctuation of these currents and the mechanisms responsible for the changes in their characteristics during the early postcontact period were also analyzed. Our results show a postcontact transition from low- to high-efficacy transmission that appears to be related to a gradual increase in nerve-muscle adhesion. This leads to the suggestion that such a transition may reflect the establishment of specific synaptic connections.

\section{Materials and Methods}

Cell culture. Xenopus nerve-muscle culture was prepared as previously reported (Spitzer and Lamborghini, 1976; Anderson et al., 1977; Xie and Poo, 1986). Briefly, the neural tube and the associated myotomal tissuc of 1-d-old Xenopus cmbryos (stagc 20-22, Nicuwkoop and Fabcr, 1967) were dissociated in $\mathrm{Ca}^{2+}-\mathrm{Mg}^{2+}$-free saline supplemented with EDTA. The cells were plated on clean glass coverslips and were used for experiments after $24 \mathrm{hr}$ at room temperature $\left(20-22^{\circ} \mathrm{C}\right)$. The culture medium consisted of $50 \%$ (vol/vol) Leibovitz medium (GIBCO), $2 \%$ 
( $\mathrm{vol} / \mathrm{vol})$ fetal bovine serum (GIBCO) and 48\% (vol/vol) of Ringer's solution $(115 \mathrm{~mm} \mathrm{NaCl}, 2 \mathrm{~mm} \mathrm{CaCl}, 2.5 \mathrm{~mm} \mathrm{KCl}, 10 \mathrm{~mm}$ Hepes, $\mathrm{pH}$ 7.3). Mononucleated spherical myocytes (myoballs) were used in the experiment of manipulated contacts. Myoballs were first loosened from the attachment to the glass substratum by "rolling" the cells across the substratum surface with a heat-polished micropipet. This loosening of attachment allowed the cell to be lifted up from the substratum when the whole-cell clamp electrode was later attached to the cell and used for cell translocation.

Electrophysiology. Synaptic currents were recorded in the muscle cell by the whole-cell patch-clamp method (Hamill et al., 1981). Recordings were made at room temperature in culture medium and the solution inside the recording pipet contained $82 \mathrm{mM} \mathrm{KCl}, 35 \mathrm{~mm} \mathrm{KOH}, 1 \mathrm{~mm}$ $\mathrm{CaCl}_{2}, 1 \mathrm{mM} \mathrm{MgCl}, 11 \mathrm{~mm}$ EGTA, and $5 \mathrm{~mm}$ Hepes buffer (pH 7.2). In a typical recording, the muscle cell was voltage-clamped at the resting membrane potential and the membrane currents were monitored by a patch-clamp amplifier (List EPC-7, Medical Systems, Greenvale, NY). The data were stored on magnetic tape (Racal S4) for later playback onto a storage oscilloscope (Textronic 5113) or an oscillographic recorder (Gould 2200). For evoking synaptic currents, the neuronal soma was stimulated extracellularly with a heat-polished glass micropipet (tip openings $2-4 \mu \mathrm{m}$ ) filled with Ringer's solution. For suprathreshold stimulation, a square current pulse $0.5-1 \mathrm{msec}$ in duration and $2-4 \mu \mathrm{A}$ in amplitude was applied. Intracellular recording at the soma has shown that such a stimulus consistently induces a single action potential in the neuron.

Quantal analysis. The conventional, and arguably the most convincing, approaches to quantal analysis of transmitter release assume that one pool of quanta gives rise to the evoked events and that all spontaneous events result from the release of a single quantal unit from that same pool. The amplitude histogram of the spontaneous events and a few parameters from the amplitude histogram of the evoked events, e.g., failure rate, coefficient of variation, and mean, are used to predict the shape of the entire evoked amplitude histogram (del Castillo and Katz, 1954; Boyd and Martin, 1956; Martin, 1977). In order to attain statistical significance with these methods, there must be a low mean quantal content, a high failure rate and a relatively large number of events. In addition, the methods depend upon a Gaussian distribution of spontaneous event amplitudes, as is usually observed. When the conditions do not meet the requirements of these methods, other approaches can be taken to test for the existence of a quantal mechanism.

In the present study, we have developed a new method called minimization of mean relative variance (MMRV), which can be used in situations where the above conditions for traditional methods are absent. Instead of using a spontaneous event histogram to construct a theoretical evoked event histogram, MMRV examines the entire evoked histogram to see whether there exists a theoretical quantal size and a theoretical statistics (Poisson, binomial, or Gaussian) that can explain the observed data. The process is computationally more intensive than the conventional methods. However, since the entire histogram of the evoked events (not just a few parameters) is examined to see if a quantal mechanism will fit, the method is more sensitive than the conventional ones and can be used is situations where there is a relatively low number of evoked events.

The procedure involves the following steps: An arbitrary quantal size (QS) is chosen. The amplitude histogram of the evoked response is "quantized," i.e., all events from 0 to $0.5 \mathrm{QS}$ are considered failures, events from 0.5 to $1.5 \mathrm{QS}$ are considered to have resulted from the release of one quantum, events from 1.5 to $2.5 \mathrm{QS}$ from 2 quanta, and so on. This yields a discrete histogram with values only at $0,1,2$, etc., quanta. The mean quantal content is then calculated. Theoretical Poisson, binomial, and Gaussian distributions with the same mean and normalization are constructed. The fit of each theoretical distribution to the "quantized" histogram of the evoked response is assessed by calculating the mean relative variance (MRV) between the two.

The MRV is calculated as follows. Let $m_{i}$ be the number of events experimentally observed that correspond to release of $i$ quanta, as indicated by the "quantized" histogram; and let $n_{i}$ be the theoretically predicted number of events releasing $i$ quanta when a total number of $N$ trials are performed. For a large set of experiments, each with $N$ trials of evoked responses, the observed number $m_{i}$ will scatter with a Gaussian distribution around the theoretical value $n_{i}$, with a variance given by

variance in $m_{i}=n_{i}\left(N-n_{i}\right) / N$.
For each experiment with total $N$ trials, the significance of the difference between the experimentally observed $m_{i}$ and theoretical value $n_{i}$ can be assessed by taking the square of the difference between these 2 numbers, weighted against the expected variance in the $m_{i}$ distribution. For each $i$, we define a relative variance by the formula

$$
R V_{i}=\left(n_{i}-m_{i}\right)^{2} / \text { variance in } m_{i} \text {. }
$$

The larger $R V_{i}$, the more significant is the difference between the observed $m_{i}$ and theoretical predicted $n_{i} . R V_{i}$ thus evaluates how well one bin in the "quantized" histogram fits the theory. To evaluate the entire histogram, MRV is defined as

$$
\mathrm{MRV}=\sum_{i=0}^{M} R V_{i} / M,
$$

where $M$ is the number of releasable quanta. Since $M$ is unknown, it suffices in actual calculation to use a value larger than the maximum quantal content observed in the set of evoked events. For each statistics and QS chosen, a value of MRV is obtained. The procedure is repeated for a series of QS. The best-fit QS is determined by a minimun in MRV. The existence of a single sharp minimum supports the idea that the release is quantal and is governed by the statistics tested. This method, when applied to the original evoked response data of del Castillo and Katz (1954) and of Boyd and Martin (1956), successfully predicted a QS that matched the observed spontaneous events.

\section{Results}

\section{Spontaneous synaptic activity}

Two types of nerve-muscle contacts were studied in the present work: Natural contacts that resulted from the random encounter of muscle cells by growing neurites and manipulated contacts that were produced by physically manipulating spherical muscle cells (myoballs) into close proximity or into direct contact with co-cultured neurons. We found that the characteristics of spontaneous synaptic activity at these 2 types of contacts were very similar, suggesting that the manipulated contact serves as a good model system for studying synaptogenesis.

Figure 1 shows an example of the recording of spontaneous synaptic activity during the first $20 \mathrm{~min}$ of a natural contact. Whole-cell recording was made on a spindle-shaped muscle cell when the latter was about to be contacted by the filopodia of a growth cone. The muscle cell was voltage-clamped at $-70 \mathrm{mV}$, and the membrane current was recorded for a period of about $20 \mathrm{~min}$. Soon after the onset of recording, inward pulsatile currents were observed in the muscle cell. These currents increased gradually in frequency and average amplitude. An example of a manipulated contact is shown in Figure 2. Whole-cell voltage clamp recording began before the myoball was manipulated into contact with the growth cone of an isolated neuron. Immediately after the contact, pulsatile inward currents as those seen in the natural contact were observed. These currents were totally abolished by bath application of curare or $\alpha$-bungarotoxin but were unaffected by tetrodotoxin (Fig. 2C; Xie and Poo, 1986), suggesting that they were due to spontaneous pulsatile release of $\mathrm{ACh}$ from the neurite in the absence of nerve impulses. In the present work, we refer to these contact-induced currents as spontaneous synaptic currents (SSCs).

Figure 3 shows histograms of SSC amplitude distributions for 2 postcontact periods marked by the dashed lines in Figures 1 and 2. These histograms indicate that there was an increase in the frequency and the mean amplitude of the SSCs, at both natural and manipulated contacts. Table 1 presents data collected during 2 postcontact periods (the time of first appearance of the SSC was defined as time 0 ) for 2 natural and 6 manipulated contacts. There was a clcar trend in the postcontact changes of the SSCs. We observed a consistent increase in SSC frequency 

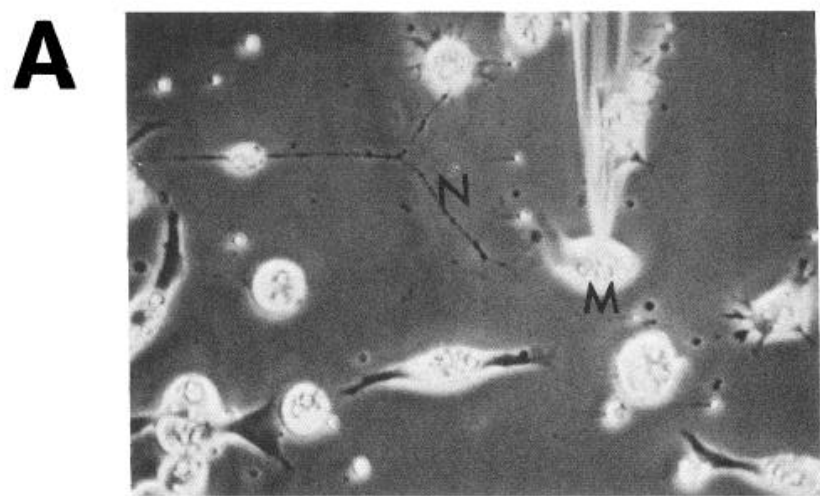
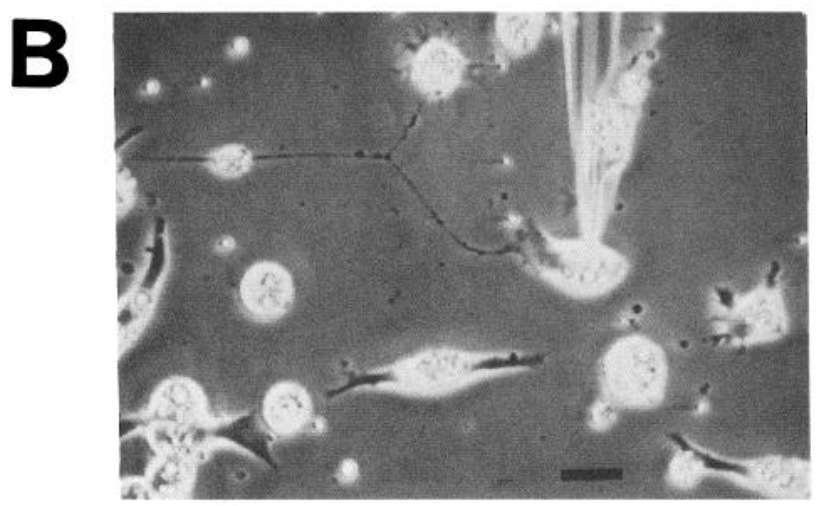

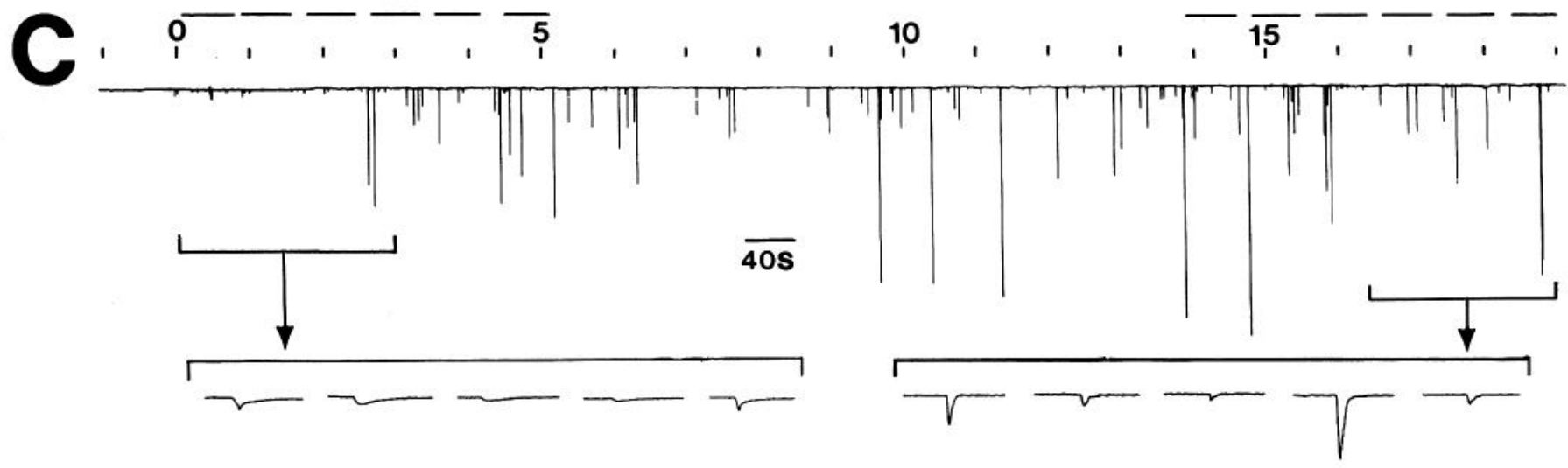

D
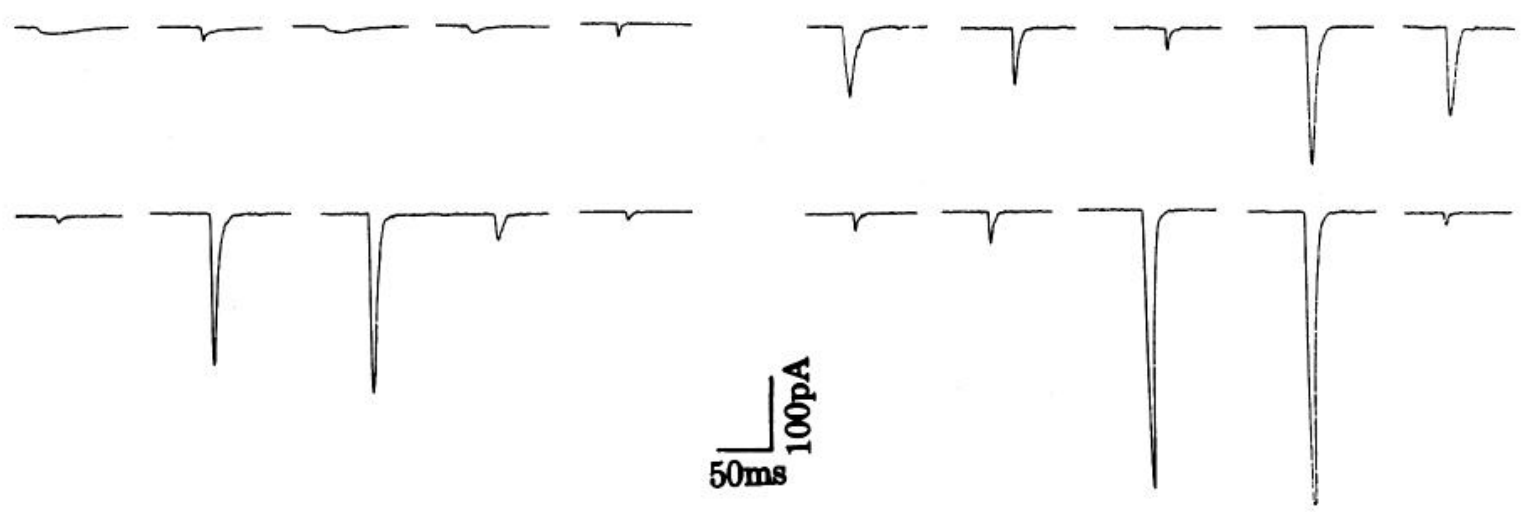

Figure 1. Spontaneous synaptic activities during the first $20 \mathrm{~min}$ of a natural neurite-muscle contact in a 1-d-old Xenopus culture. $A$ and $B$, Phasecontrast photographs of the growing neurite $(N)$ and spindle-shaped myocyte $(M)$, taken at the beginning and the end of electrical recording, respectively. Scale bar, $25 \mu \mathrm{m}$. $C$, Continuous tracing shows membrane currents through the myocyte under whole-cell voltage-clamp condition $\left(E_{m}=-70 \mathrm{mV}\right.$, inward current downward, filtered at $\left.150 \mathrm{~Hz}\right)$ for a duration of about $20 \mathrm{~min}$, starting prior to the visible contact of the growth cone with the myocyte. Numbered marks indicate time (in min) after the onset of recording. $D$, The first and last 15 current pulses observed during the $20 \mathrm{~min}$ period are shown at higher time-resolution (filtered at $1.2 \mathrm{kHz}$ ).

in all cases and a significant increase in the mean amplitude $(p$ $<0.01$ or lower, $t$ test) for 1 out of 2 natural contacts and 4 out of 6 manipulated contacts. Either or both of the following changes could account for the increase in mean SSC amplitude: an increase in amplitude for all currents and the appearance of a disproportionally larger fraction of SSCs of higher amplitudes. No direct evidence is available to distinguish between these possibilities. We have also examined the changes in the average rise time (time-to-peak) of the SSCs during the first $20 \mathrm{~min}$ following the contact. No consistent change was observed in either the natural or manipulated contacts (see Fig. $3 C, F$ ).

\section{Contacts with different regions of the neuron}

When a voltage-clamped myoball was manipulated into contact with a co-cultured neuron at different regions along the neurite process and at the soma, SSCs similar to those observed at the 

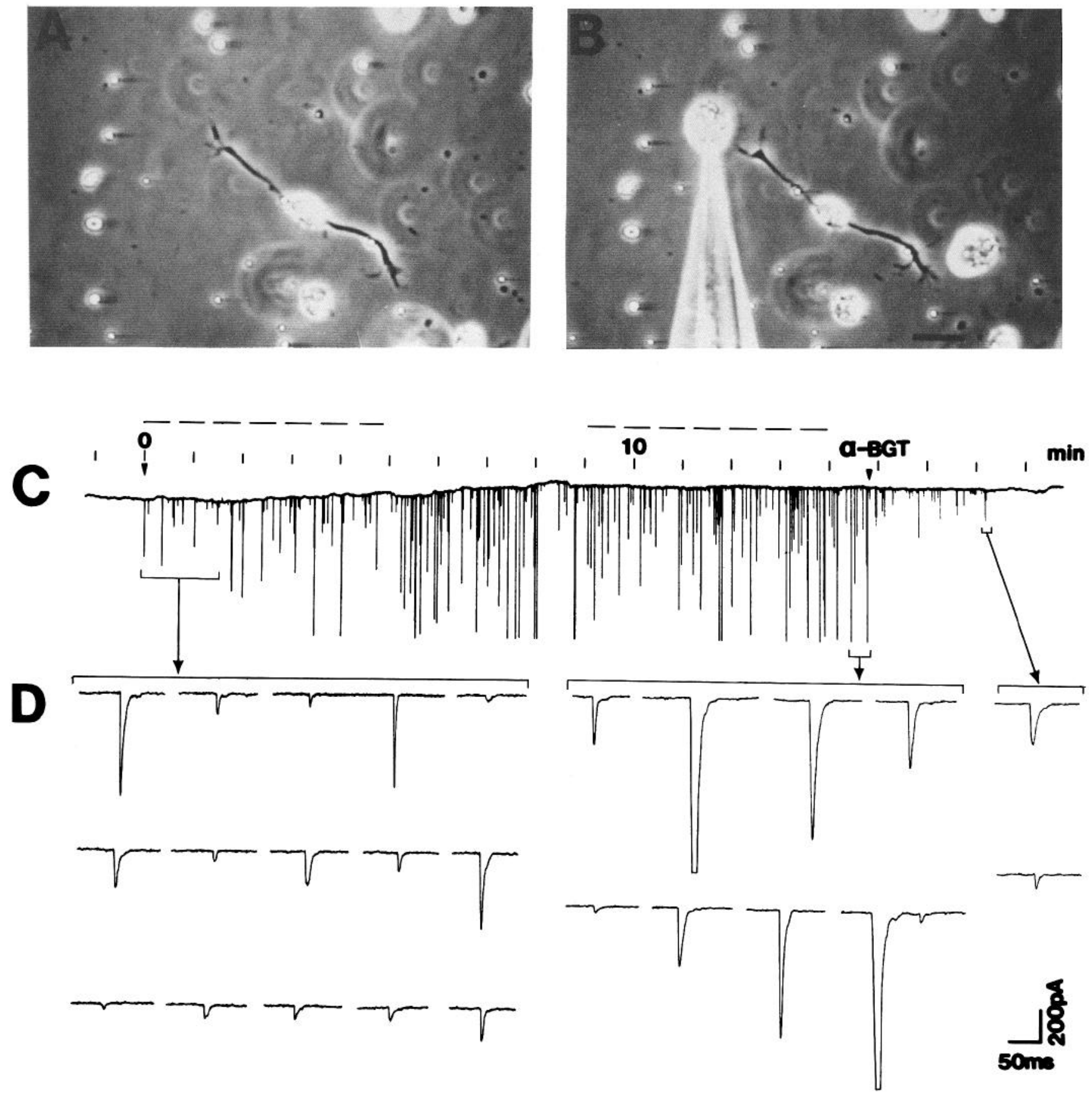

Figure 2. Spontaneous synaptic activities during the first $20 \mathrm{~min}$ of a manipulated neurite-muscle contact in a 1-d-old Xenopus culture. $A$ and $B$. Phase-contrast photographs of the cells before and after the positioning of the spherical myocyte $(M)$ at the growth cone of the neurite. Scale bar, $30 \mu \mathrm{m}$. C, Continuous tracing shows myocyte membrane current recorded under the same voltage-clamp conditions as described in Figure 1 . The recording started prior to the cell manipulation. The time of neurite-myocyte contact is marked as 0 . The pulsatile currents were abolished after local perfusion with $\alpha$-bungarotoxin $(\alpha-B G T, 10 \mu \mathrm{g} / \mathrm{ml}) . D$, Samples of currents are shown at higher time-resolution.

growth cone were often found. Release of $\mathrm{ACh}$ from soma of Xenopus spinal neurons has been observed previously by similar cell manipulation methods (Chow and Poo, 1985). In the present study, we made further comparison between the releases at different regions of the same neuron, using the same myoball for triggering the release. Figure 4 shows 2 sets of recordings for contacts made between the same myoball with different regions of the same neuron. We made paired comparison of SSC activ- ities (during the first $5 \mathrm{~min}$ postcontact period) for contacts at growth cone versus soma, and at growth cone versus neurite process. In these experiments, we attempted to exert similar pressure between the myoball and the neuron (see next section), although no quantitative assay of the pressure and/or contact area was made. In 2 out of 3 cases studied, the mean SSC amplitude was significantly higher at the growth cone than at the soma $(p<0.001)$. The frequency of SSCs was higher at the 
NATURAL
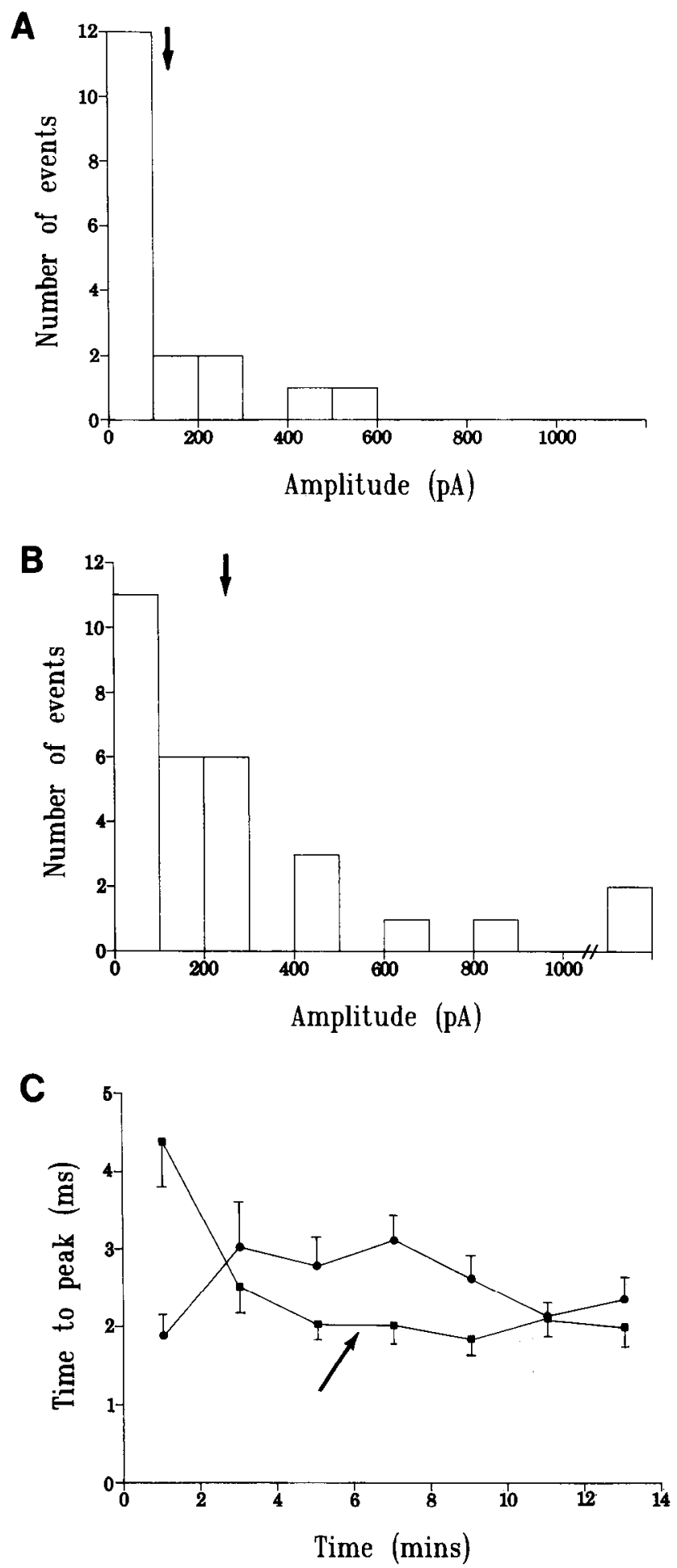

\section{MANIPULATED}
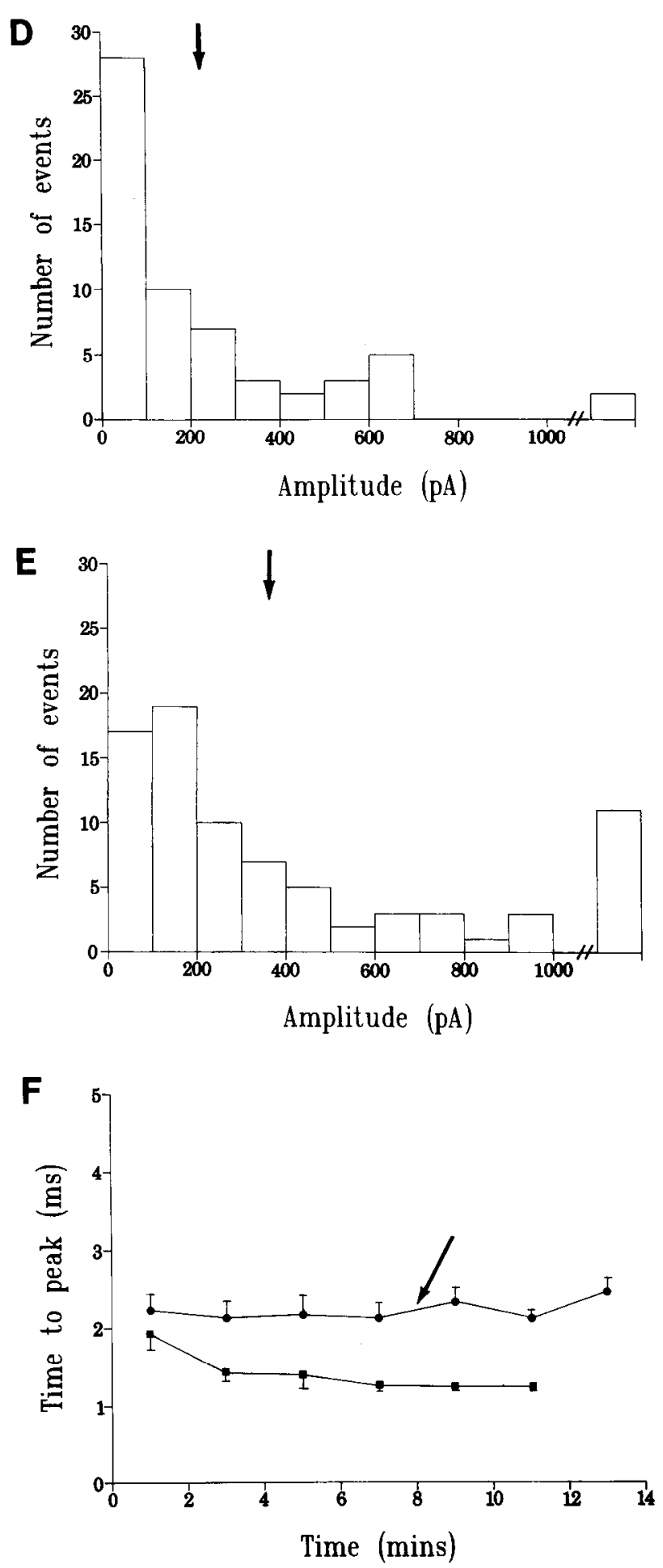

Figure 3. Comparison of spontaneous synaptic currents at natural and manipulated contacts. Data obtained in experiments shown in Figures 1 and 2 were analyzed for the amplitude and time course of synaptic currents. $A, B, D$, and $E$, Histograms of current amplitude for 2 postcontact periods (5 min each, marked by dashed lines in Figs. 1 and 2), respectively, for natural and manipulated contacts. Panels $A$ and $D$ are for the early periods and $B$ and $E$ are for the later periods. Arrows above the histograms mark the mean amplitude. $C$ and $F$, Rise time (time-to-peak) of all spontaneous synaptic currents plotted as a function of postcontact time, respectively, for 2 natural and 2 manipulated contacts. Arrows mark the data from the contacts described in Figures 1 and 2. Data were grouped into 2 min bins, and the error bars represent SE. In the data represented by the squares $(\square)$, the difference between the first and the last data points is statistically significant $(t$ test, $p<0.01)$. 

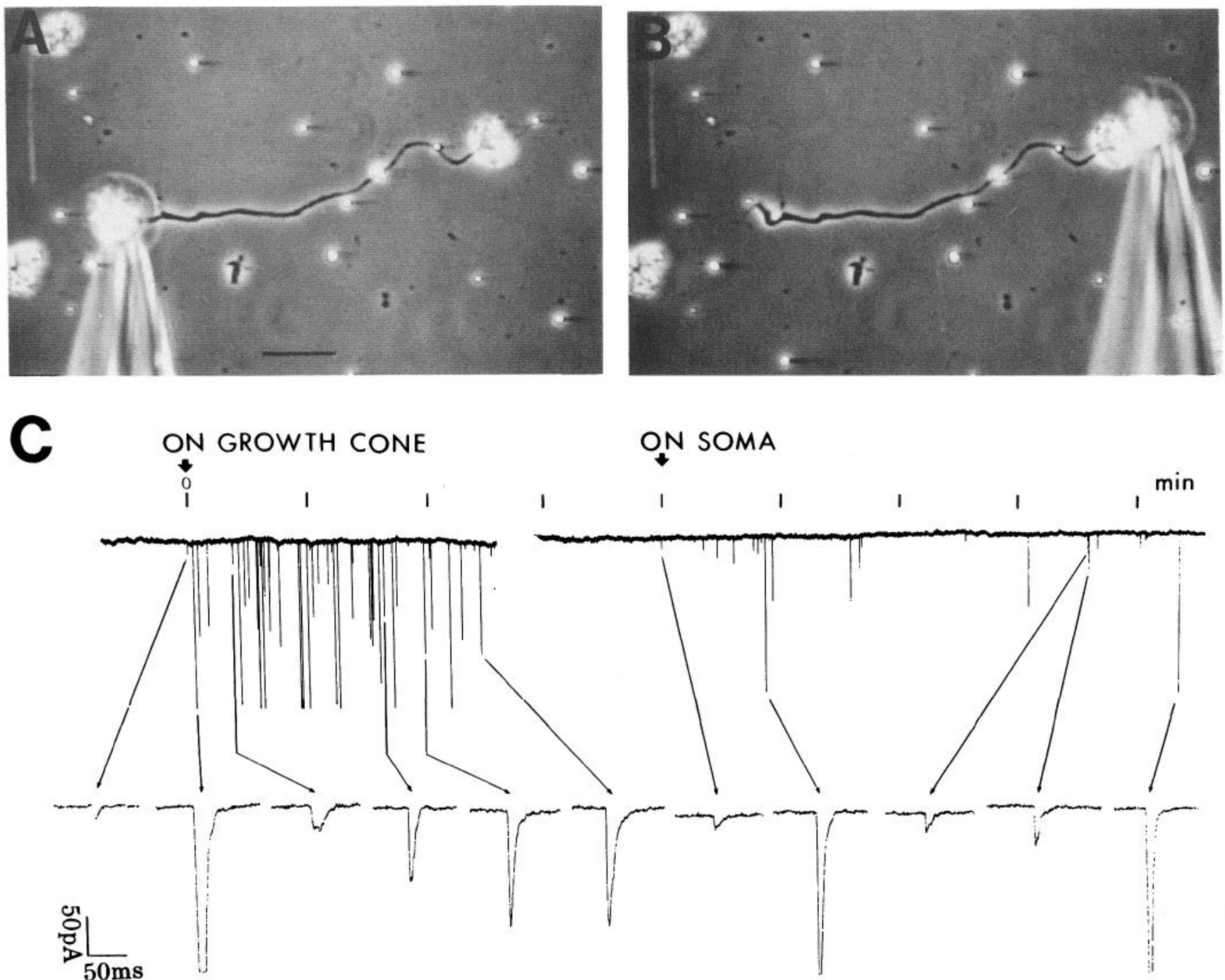

Figure 4. Induction of $\mathrm{ACh}$ release by muscle contacts with different regions of the neuron. The same voltage-clamped myoball was manipulated into contact sequentially with the growth cone and the soma of an isolated neuron. $A$ and $B$, Phase-contrast images of the cells during the recording. Scale bar, $30 \mu \mathrm{m}$. $C$, Continuous traces are recordings of membrane currents showing spontaneous synaptic currents recorded at the growth cone and the soma, respectively. Samples of these currents are shown at the bottom at higher time resolution. $D$, Data from another cell pair. Currents were recorded sequentially for contacts by the same myoball at the growth cone, the neurite, and soma.

growth cone in all 3 cases (percentage difference: 12, 25, 214). For growth cone versus neurite comparison, no significant differences in SSC amplitude were found in the 4 cases studied $(p$ $>0.05$ ). However, the SSC frequency was higher at the growth cone in all 4 cases (percentage difference: $9,56,116,178$ ). The capability of ACh release at all regions of the neuron indicates that the interacting neuronal surface molecule(s), the cytoplasmic ACh pool, and the secretory mechanism for ACh release are not exclusively localized in these cultured neurons. On the other hand, the difference in SSC amplitude does suggest that the muscle's induction of ACh release and/or the synaptic transmission at the soma is less efficacious than that of the rest of the neuron. The higher frequency of spontaneous release at the growth cone may reflect a larger available surface area for muscle contact due to the presence of many filapodial protrusions.

\section{Repeated contacts with the same growth cone}

To test the reproducibility in the induction of spontaneous ACh release, we made repeated manipulated contacts between the same growth cone and myoball. We found that, while similar SSCs were observed in each contact, the frequency and the mean amplitude of the currents varied according to the pressure applied to the myoball-neurite contact by the micropipet. Figure $5 A$ shows 2 successive trials in which contacts were made (for 5 min each) between the same myoball and growth cone, with different pressures. Amplitude histograms (Fig. $5 B$ ) for the SSCs recorded during these 2 trials indicate that there were more SSCs of higher amplitudes when higher pressure was applied to the myoball. The mean amplitudes of the SSCs were statistically different $(p<0.001, t$ test). For 4 different nerve-muscle pairs 


\section{GROWTH CONE}
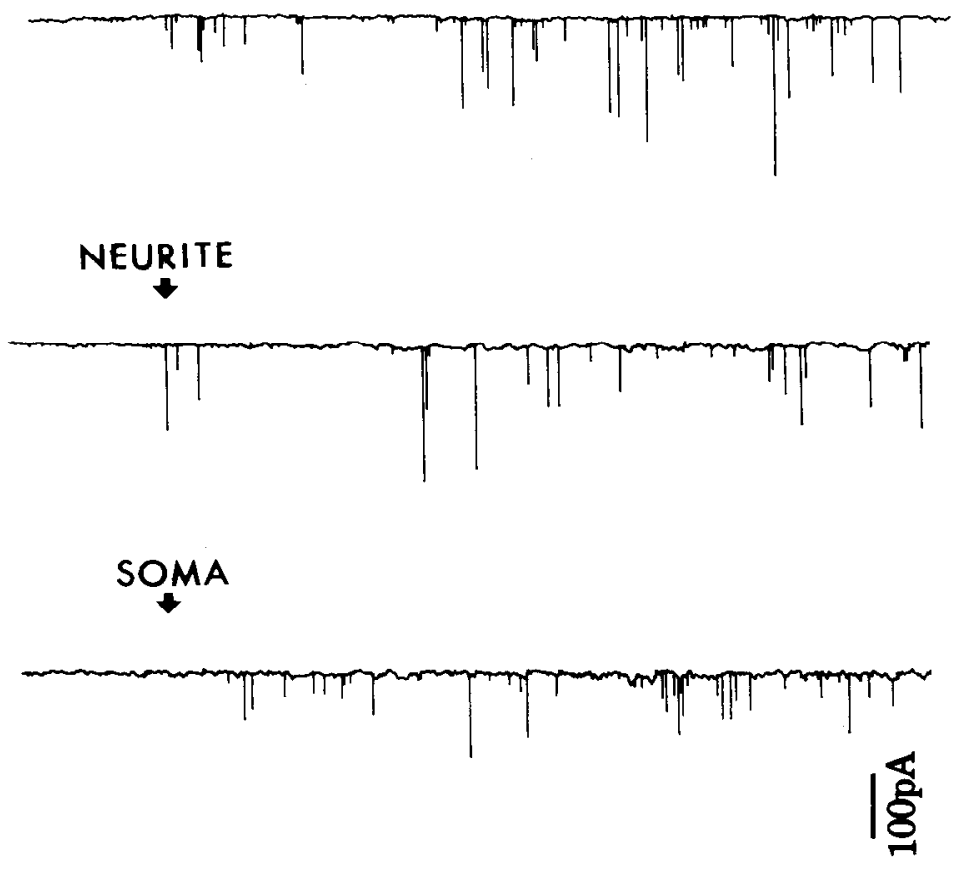

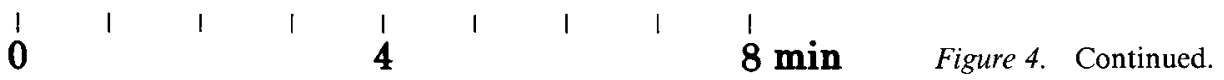

examined, the frequency of SSCs for high-pressure contacts was higher in all cases (percentage difference: $17,45,87,149$ ). The mean amplitude was significantly higher in 3 out of 4 cases $(p$ $<0.02$ or lower). This pressure-dependent phenomenon was observed regardless of whether the high-pressure trial preceded or followed the low-pressure trial. In the example shown in Figure $5 A$, increased pressure also caused a significant decrease in the rise time of the SSCs from $2.8 \pm 0.9$ to $2.4 \pm 0.7 \mathrm{msec}$ (mean $\pm \mathrm{SD}, N=94$ and $110, p<0.001$ ). A similar effect on the frequency and amplitude of the SSCs was also found when the myoball was pressed down onto the growth cone by advancing the recording electrode on the muscle cell in $2 \mu \mathrm{m}$ steps (Fig. 5C). Taken together, these findings are consistent with the idea that pressing the myoball harder on the neurite results in

Table 1. Postcontact increase in spontaneous synaptic activity

\begin{tabular}{|c|c|c|c|c|c|c|}
\hline \multirow[b]{2}{*}{$\begin{array}{l}\text { Experi- } \\
\text { ment } \\
\text { number }\end{array}$} & \multicolumn{2}{|l|}{$0-5$} & \multicolumn{2}{|c|}{$15-20 \mathrm{~min}$} & \multicolumn{2}{|c|}{ Percentage increase $^{a} \mathrm{~min}$} \\
\hline & $\begin{array}{l}\text { Number } \\
\text { of } \\
\text { events }\end{array}$ & $\begin{array}{l}\text { Mean } \\
\text { amplitude } \\
(\mathrm{pA})\end{array}$ & $\begin{array}{l}\text { Number } \\
\text { of } \\
\text { events }\end{array}$ & $\begin{array}{l}\text { Mean } \\
\text { amplitude } \\
\text { (pA) }\end{array}$ & $\begin{array}{l}\text { Number } \\
\text { of } \\
\text { events }\end{array}$ & $\begin{array}{l}\text { Mean } \\
\text { amplitude }\end{array}$ \\
\hline \multicolumn{7}{|c|}{ Natural contact } \\
\hline 1 & 18 & $133 \pm 37^{b}$ & 30 & $254 \pm 51$ & 67 & 91 (n.s.) \\
\hline 2 & 30 & $61 \pm 21$ & 70 & $173 \pm 24$ & 133 & $184(p<0.001)$ \\
\hline \multicolumn{7}{|c|}{ Manipulated contact } \\
\hline 1 & 21 & $19 \pm 5$ & 71 & $46 \pm 8$ & 238 & $142(p<0.01)$ \\
\hline 2 & 110 & $99 \pm 8$ & 200 & $297 \pm 17$ & 82 & $200(p<0.001)$ \\
\hline 3 & 34 & $26 \pm 8$ & 73 & $32 \pm 6$ & 115 & 23 (n.s.) \\
\hline 4 & 25 & $12 \pm 3$ & 284 & $95 \pm 4$ & 1036 & $692(p<0.001)$ \\
\hline 5 & 18 & $74 \pm 20$ & 57 & $90 \pm 12$ & 217 & 22 (n.s.) \\
\hline 6 & 60 & $228+28$ & 81 & $365+36$ & 35 & $60(p<0.01)$ \\
\hline
\end{tabular}

a "Percentage increase" refers to the increase between the data obtained at 0-5 min and 15-20 min expressed as a percentage of the value at $0-5 \mathrm{~min}$.

${ }^{b}$ Mean \pm SEM.

' Statistical analysis using the Student's $t$ test. n.s., $p>0.05$. 
Figure 5. Repeated contacts between the same growth cone and myoball. $A$, Two contacts were made for a period of about $5 \mathrm{~min}$ each. The recording pipet was pressed slightly harder on the myoball during the first contact. Continuous tracing shows the membrane current as described before. $O N$ marks the time of myoball contact with the growth cone, and $O F F$ marks the time of myoball removal from the growth cone. Currents observed when the myoball was away from the growth cone was due to residue growth cone elements carried away by the myoball. $B$, Histograms of current amplitudes for 2 separate contacts shown in $A$. Note the appearance of higher-amplitude currents in the contact with higher pressure. $C$, Stepwise increase of pressure at a myoball-growth cone contact. A voltage-clamped myoball was first gently placed on top of an isolated growth cone (at time marked by the first arrow), and the recording pipet on the myoball was then advanced downward in 2 steps ( $2 \mu \mathrm{m}$ each, 6 min apart) at the times marked by the second and third arrows, resulting in stepwise increments in the pressure on the myoball.
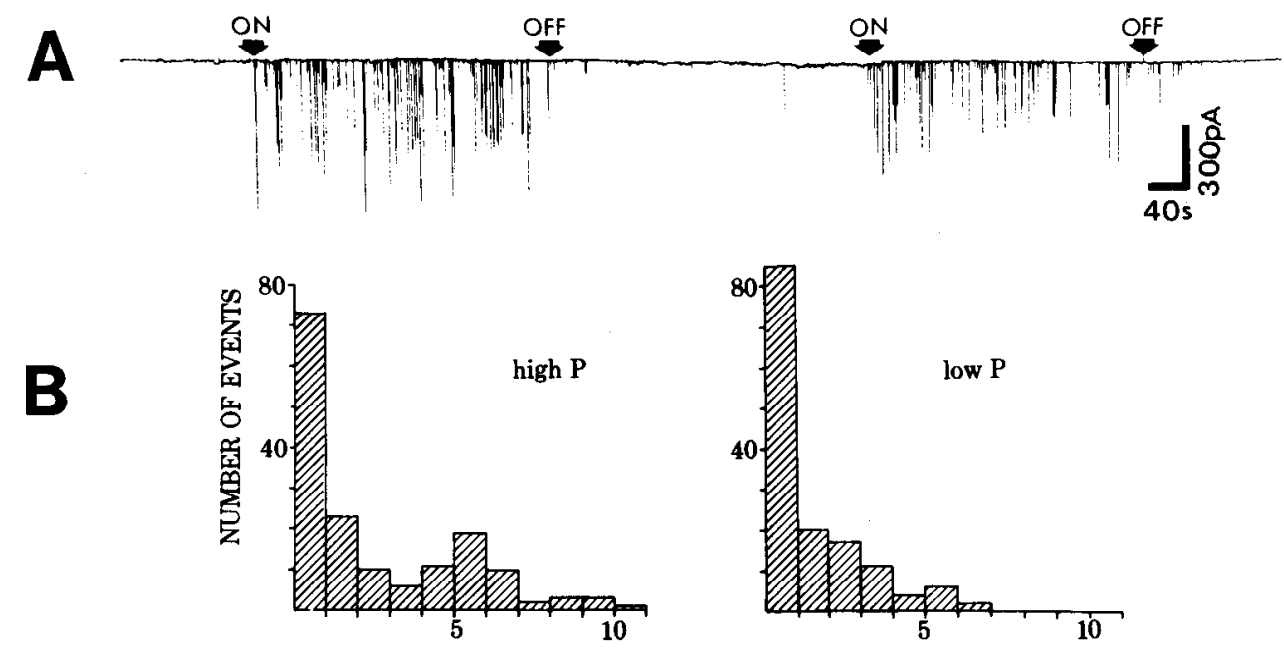

AMPLITUDE OF CURRENT PUISES (100pA)

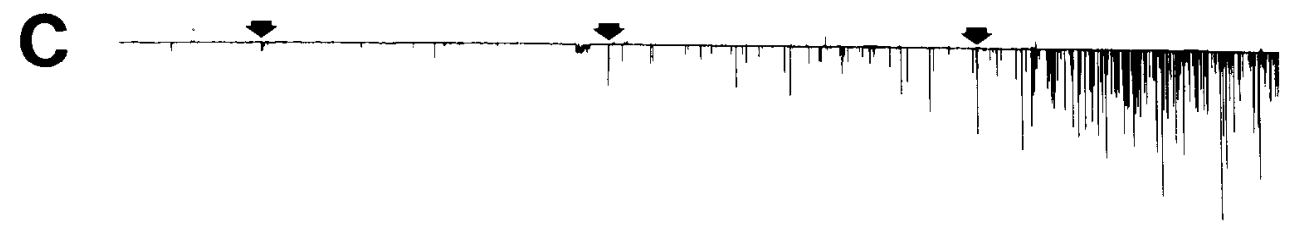

a closer and/or larger area of membrane-membrane apposition between the nerve and muscle cells, leading to an increased induction of $\mathrm{ACh}$ release and a higher amplitude of the $\mathrm{ACh}$ response. It may be noted that the amplitude histogram for the high-pressure trial resembles that observed in both natural and manipulated contacts at later periods (Fig. 3, $B, E$ ). This suggests that high pressure or prolonged contact results in the development of a similar membrane-membrane interaction between the nerve and muscle cells.

\section{Postcontact increase in nerve-muscle adhesion}

During the study of the repeated contacts, we noticed that when a myoball was removed from the growth cone, part of the growth cone was often carricd away by the myoball and long strings of membranous filaments formed between the myoball and growth cone. Removing the myoball after longer contact periods frequently led to detachment of the growth cone or even the entire neurite from the culture substratum. (The duration of each of the repeated contacts described in the above section was thus kept to 5 min or less.) Figure 6, $A-C$, illustrates 3 separate cases of graded adhesion between the myoball and the growth cone. Four qualitative grades of adhesion were defined: grade 0 , no visible nerve-muscle attachment seen during the separation; grade 1, membranous filament seen connecting the myoball and the neurite but the main "palm" of the growth cone showing no visible deformation (Fig. 6A); grade 2, myoball removal leading to deformation of the growth cone (Fig. $6 \mathrm{~B}$ ); and grade 3 , myoball removal leading to detachment of the growth cone or neurite from the culture substratum (Fig. 6C). To determine the time course for the development of this nerve-muscle adhesion, 2 groups of myoball-growth cone contacts were made, and the myoballs were removed from the growth cones after a period of either 1.5 or $15 \mathrm{~min}$, respectively. The adhesion was graded according to the above criteria. Figure $6 D$ depicts the histograms of adhesion grades obtained in this experiment. It is apparent that low-grade adhesion can quickly develop after the contact. However, the adhesion increases still further during the first $10-$ 15 min. This postcontact increase in adhesion may be responsible for the changes in the synaptic current properties (see Discussion).

\section{Origin of variation in SSC amplitude}

While the postcontact increase in the nerve-muscle membrane apposition may account for the gradual increase in the frequency and the mean amplitude of the SSCs, it is not clear what accounts for the marked variation in the amplitude of individual SSCs at a given time. This amplitude variation could be due to a number of causes: (1) Variation in the amount of ACh contained in each "package" of release that induces a SSC, (2) variation in the local $\mathrm{ACh}$ receptor density in the muscle membrane, (3) differences in the distance between the release site on the neurite and the muscle membrane, and (4) differences in the rate of degradation or diffusion-limited dissipation of $\mathrm{ACh}$ at different release sites. The following experiments were carried out to test these possibilities.

We first examined the variation of $\mathrm{ACh}$ receptor density over the surface of the muscle membrane. Identical pulses of ACh were iontophoretically applied to the muscle surface at random points along the half-perimeter of the isolated myoball, and ACh-induced membrane currents were recorded by the wholecell, voltage-clamp method described above. The tip of the iontophoretic pipet was placed in contact with the muscle surface in order to simulate close apposition of the neurite and muscle membrane. For each myoball, the iontophoretic current was set 

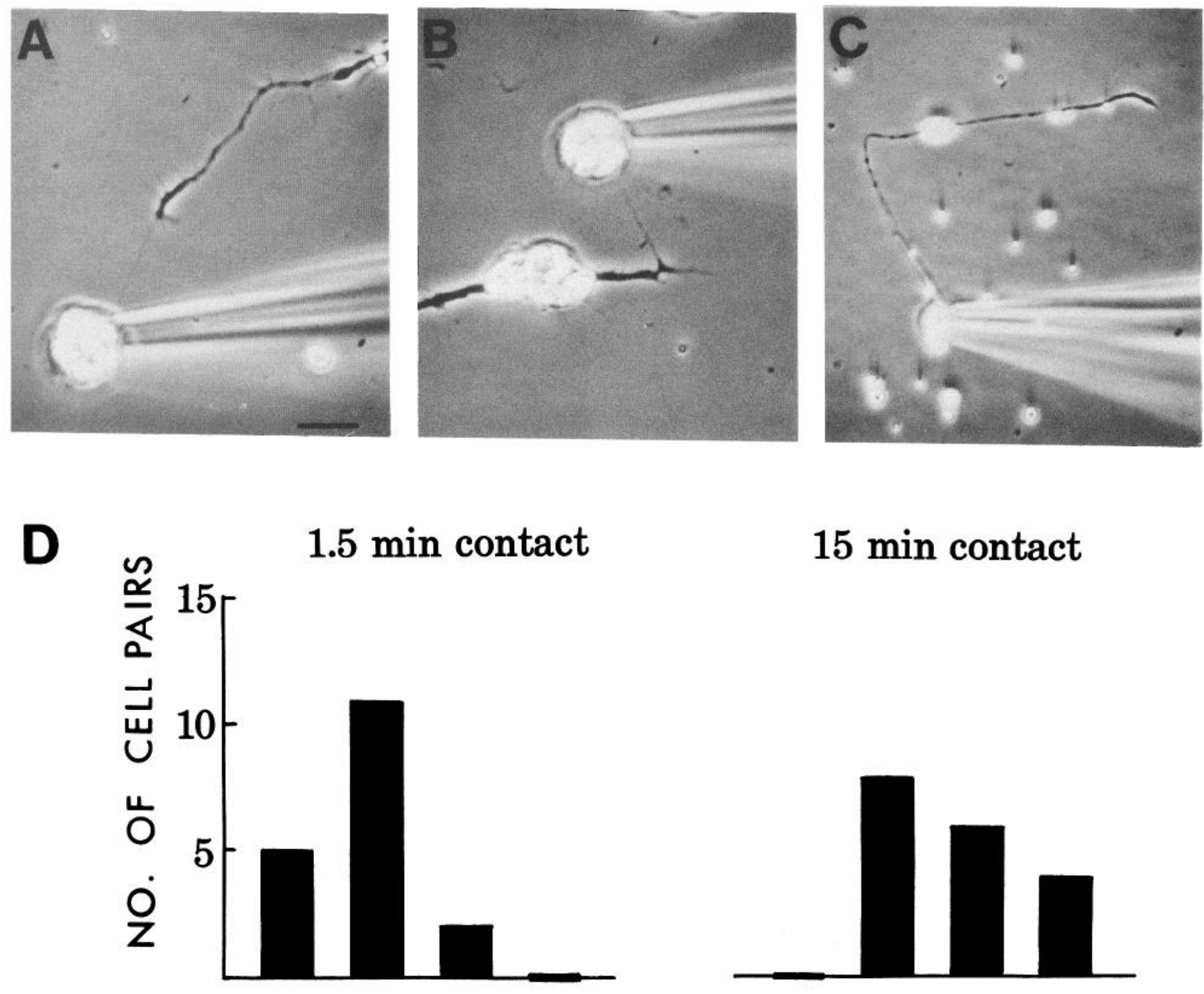

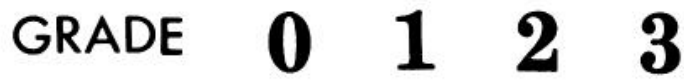

Figure 6. Postcontact increase of neurite-muscle adhesion. Myoballs were manipulated into contact with growth cones of co-cultured spinal neurons for different periods and then pulled away from the growth cones. The adhesion between the cells was estimated by a qualitative grading method (see text for details). $A-C$, Light micrographs illustrating grade 1-3 adhesion, respectively. Scale bar, $30 \mu \mathrm{m}$. Note the filamentous structure between the growth cone and the myoball in $A$, the distortion of the growth cone in $B$, and lifting of the neurite (out of focus) in $C$. $D$, Histograms of adhesion grades for 2 groups of neurite-muscle contacts, with the duration of contact 1.5 or 15 min, respectively. Note grade 3 adhesion (detachment of neurite during separation) was seen only in the group of $15 \mathrm{~min}$ contacts.

at a fixed level that produced ACh-induced currents with amplitudes and rise times within the range covered by typical SSCs. Measurements were taken for 15-30 points on each myoball, and 5 myoballs from 2 separate cultures were used. The SD of the current variation on 5 different myoballs ranges from 14 to $26 \%$ of the mean amplitude. The deviation could be due to variation in the $\mathrm{ACh}$ receptor density, as well as in the proximity of the iontophoretic pipet from the muscle surface. Even so, the variation of these $\mathrm{ACh}$-induced currents was much smaller than that of the SSCs: In the 2 cases of nerve-muscle contact shown in Figure 3, the SDs of SSCs were between 93 and $111 \%$ of the mean amplitudes for the 4 histograms. The present iontophoretic method may not be sensitive enough to detect ACh receptor clusters of submicron sizes, since the ejected ACh pulse covers a range of a few microns within the rise time of the membrane current. However, the surface area sampled by the iontophoretic and nerve-released $\mathrm{ACh}$ is similar, since they have similar rise times and amplitudes. Microclusters unresolved by the our iontophoretic pulses will not contribute substantially to SSC amplitude variation.

If $\mathrm{ACh}$ receptor density is relatively uniform on the surface of isolated myoball, could the neurite-contact cause varying degrees of local receptor clustering, leading to variation in SSC amplitude? This seems unlikely, since SSCs of markedly different amplitudes were observed within seconds following the contact (Xie and Poo, 1986; also Fig. 2), a time too short for even a small cluster to form at the initial contact site. Assuming the most favorable conditions for cluster formation, namely, a high lateral diffusion coefficient of $3 \times 10^{-9} \mathrm{~cm}^{2} / \mathrm{sec}(\mathrm{Poo}, 1982)$ and a perfect sink at the neurite contact site for clustering, the for- 
Figure 7. Comparison of spontaneous synaptic currents with iontophoretically induced currents. A myoball was voltage-clamped at its resting potential $(-70 \mathrm{mV})$, and currents induced by identical iontophoretically applied $\mathrm{ACh}$ pulses were recorded. The iontophoresis pipet was placed at different distances from the surface of the myoball so that the same ACh pulses induced currents of varying amplitudes. Samples of these currents for 3 different positions are shown in $A$. Note that the artifact at the beginning of each current was due to iontophoretic current application. The same myoball was then positioned into contact with a growth cone, and the spontaneous synaptic currents were recorded for a period of $10 \mathrm{~min}$. Samples of the currents are shown in $B$. Scales: $10 \mathrm{msec}, 100 \mathrm{pA}$. $C$, The time-to-peak and the amplitude of each of the iontophoretically induced currents (-0) and spontaneous synaptic currents (-O-) were plotted. Data were grouped into 5 bins according to the current amplitude. Error bars indicate SD. Note the clear trend that smaller SSCs have shorter rise times, exactly opposite to that for iontophoretically induced currents.
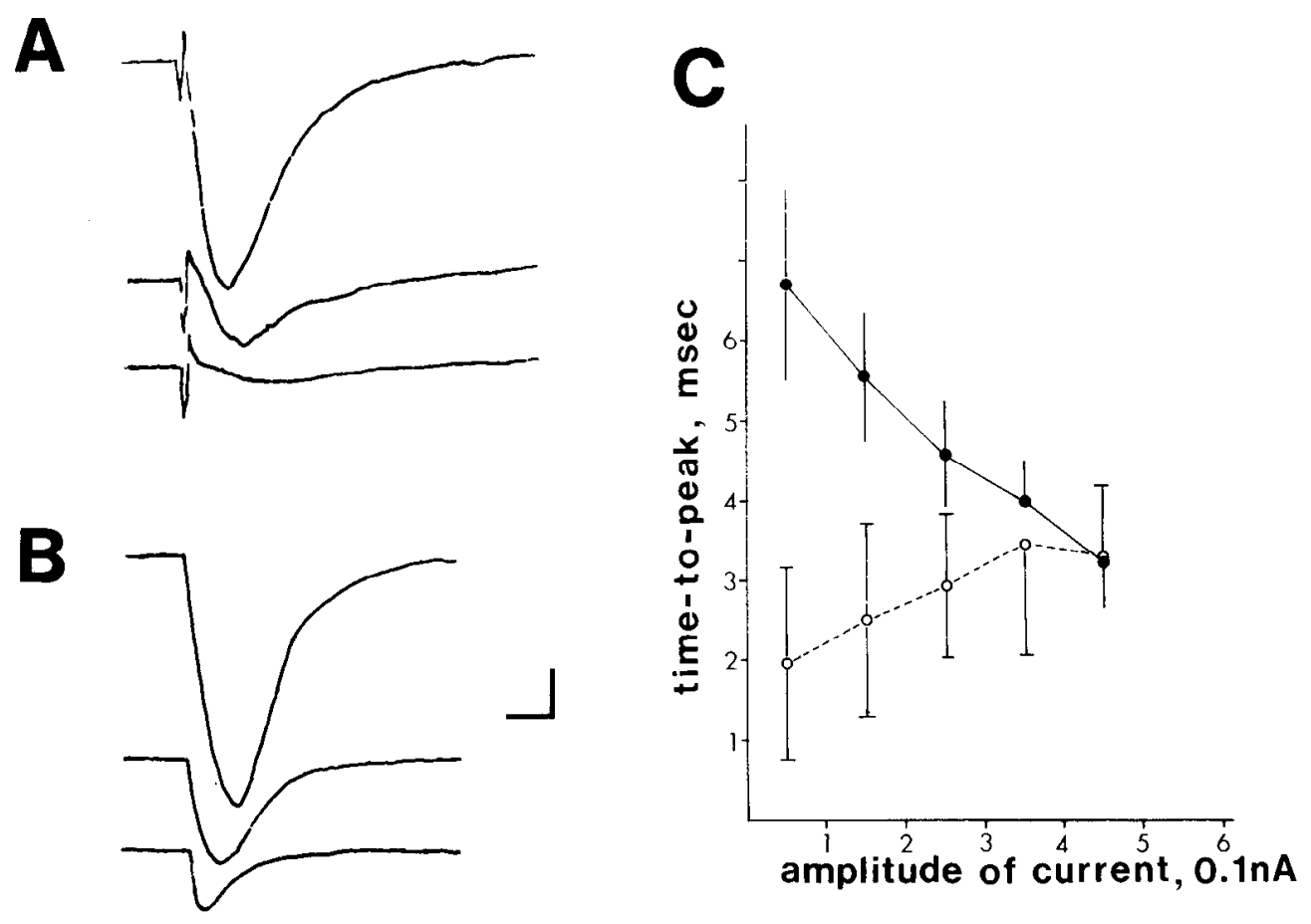

mation of a small ACh receptor cluster $1 \mu \mathrm{m}$ in diameter with a receptor density 10 -fold higher than that of the noncontact region would require a few minutes (Chao et al., 1981).

To study the effect of the distance between the release site and the muscle membrane, an $\mathrm{ACh}$ pipet was positioned at different distances from the surface of the myoball, and the membrane currents in response to identical iontophoretic $\mathrm{ACh}$ pulses were recorded. The same myoball was then manipulated into contact with a neurite and the SSCs were recorded. The amplitudes and the rise times of both iontophoresis- and neurite-induced currents were plotted and compared. The result, as shown in Figure 7, indicates that if the amplitude variation of the SSC's were due solely to the distance of the release site from the muscle membrane, the rise time for the SSC should increase as the amplitude bccomes smaller-in a manner similar to that of the currents produced by the same iontophoretic ACh pulse at different distances. Our observation showed that this was not the case: The rise times of the smaller SSCs were similar to or even shorter than those of the larger ones.

Assuming that the release sites for spontaneous release are at equal distance from the muscle membrane, the same package of released $\mathrm{ACh}$ could still produce different muscle responses if the $\mathrm{ACh}$ molecules are degraded or dissipated from the contact zone at different rates. The degradation rate depends on the concentration of cholinesterases that may be associated with each release site (Moody-Corbett et al., 1982). The diffusion of released $\mathrm{ACh}$ away from the release site could vary, depending upon the extent of membrane-membrane apposition surrounding each release site. To test the possible involvement of cholinesterases, we carried out manipulated contact experiments in the presence of 2 cholinesterase inhibitors, neostigmine $(10 \mu \mathrm{g} /$ $\mathrm{ml})$ and eserine $(20 \mu \mathrm{g} / \mathrm{ml})$. These drugs were added to the culture medium either before or after the contact, in 2 separate trials.
No significant effect of the drug treatment was observed, suggesting that cholinesterases are unlikely to be involved in determining the amplitude of the SSCs at this early stage of nervemuscle contact. The idea that close nerve-muscle membrane apposition could impede the dissipation of extracellular ACh, and hence increase the size of the postsynaptic response, has not been tested directly. It may be noted that ultrastructural studies did observe occurrence of close membrane appositions between nerve and muscle cells during the early stages of contact (Buchanan et al., 1989).

Taken together, we have 2 remaining mechanisms that may explain the amplitude variation, namely, differences in either the amount of $\mathrm{ACh}$ in each package of spontaneous release or the extent of membrane-membrane apposition surrounding each release site. The relative contributions of these 2 mechanisms remain to be studied.

\section{Impulse-evoked synaptic activity}

A physiologic definition of a functional synapse is the occurrence of the evoked synaptic current in the postsynaptic cell in response to an action potential in the innervating neuron. Since transmitter secretion from isolatcd growth cones can be evoked by action potentials (Hume et al., 1983; Sun and Poo, 1987) and embryonic muscle cells carry $\mathrm{ACh}$ receptors in their plasma membrane prior to nerve contact, we expect to see evoked responses as soon as the muscle membrane is in close proximity to the secreting growth cone. This was indeed observed in the present study. As illustrated in Figure 8, a voltage-clamped myoball was manipulated into contact with the growth cone of an isolated neuron at time marked "ON." The neuron was stimulated extracellularly at the soma at a frequency of about 0.1 $\mathrm{Hz}$ during the entire course of recording. In this and other similar recordings, we consistently observed nerve-evoked synaptic cur- 


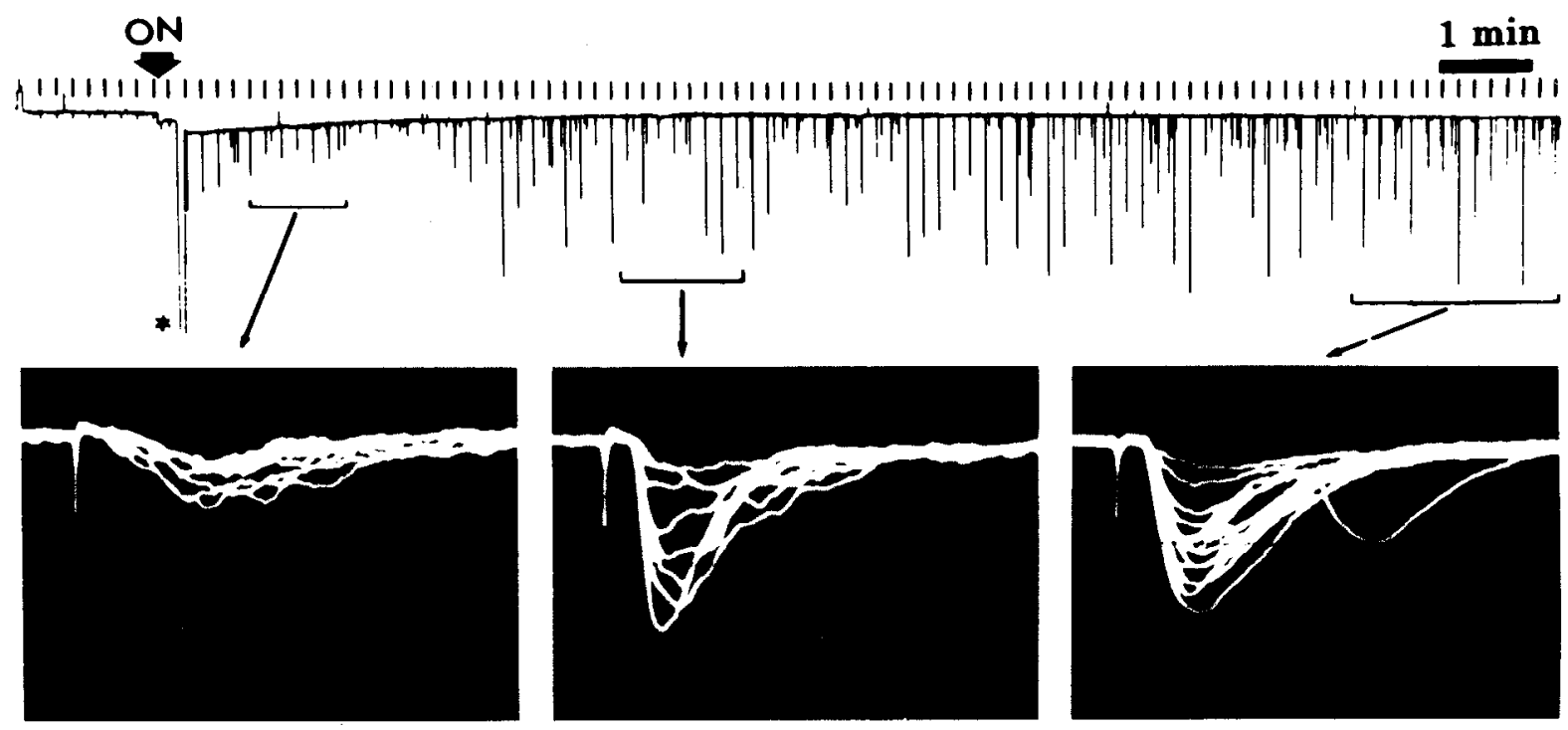

Figure 8. Evoked synaptic currents during the first $20 \mathrm{~min}$ of neurite-muscle contact. An isolated neuron was electrically stimulated at the soma to fire action potentials at a rate of about $6 / \mathrm{min}$. A voltage-clamped myoball ( $V_{m}-65 \mathrm{mV}$ ) was manipulated into contact with the growth cone of the neuron (neurite length, $150 \mu \mathrm{m}$ ), and the membrane currents were recorded continuously. Evoked synaptic currents were observed immediately following the contact (at time marked by $O N$ ), together with the spontaneous currents. The latter are visible in the recording as events that occurred between the stimuli (marked by scale lines above the current trace). Samples of evoked synaptic currents are shown at higher time resolution (filtered at $2.5 \mathrm{kHz}$ ) for 3 postcontact periods (brackets). Scales: $5 \mathrm{msec}, 500 \mathrm{pA}$. The origin of the large inward current (asterisk) at the beginning of this particular contact is unknown.

rents (ESCs) within the first minute of contact, together with the appearance of the spontaneous synaptic currents, as described in the earlier sections. Furthermore, significant postcontact changes in the ESCs were seen. Figure 9 presents data from 3 different contacts (including the one shown in Fig. 8). During the first $15 \mathrm{~min}$ postcontact period, we found in all cases a significant increase in the average amplitude and a significant reduction in the delay of onset of the ESCs following the stimulus. Similar to the case of SSCs described earlier, no consistent postcontact change was seen in the rise time (time-to-peak) of the ESCs.

\section{Quantal analysis of ESC}

During the first few hours of synaptogenesis, the amplitude of the evoked synaptic currents showed high stimulus-to-stimulus variation in physiological solutions (normal $\mathrm{Ca}^{2+}$ and $\mathrm{Mg}^{2+}$ concentrations). Does transmitter release occur in multiples of a quantal unit and the amplitude fluctuation therefore reflect differences in the number of quanta released per nerve impulse?

Intrinsic limitations in the present developing system prevented the use of conventional approaches in the quantal analysis of synaptic transmission. First, the amplitude histogram of the spontaneous events does not show a single Gaussian distribution, as is found in the mature synapse. Second, the synapse may be changing its characteristics during the period of recording. Collection of a sufficient number of events within short periods is difficult since the synapse fatigues rapidly under stimulus frequencies in excess of $1 \mathrm{~Hz}$. Finally, attempts to lower mean quantal content by treatment with low $\mathrm{Ca}^{2+}$ failed because the synapse is not viable under prolonged exposure to low $\mathrm{Ca}^{2+}$. A new method of quantal analysis-minimization of mean relative variance (MMRV) - was used in the present study (see Materials and Methods). Briefly, MMRV examines the evoked response amplitude histogram and tries various statistics (Pois- son, binomial, and Gaussian) and a series of test QSs to generate theoretical evoked histograms. The fit between these theoretical histograms and the actual data histogram is assessed by calculating MRV between the two. The QS and the statistics that generate the best fit (minimal MRV) is then determined.

Figure $10 \mathrm{~A}$ depicts data of evoked synaptic currents recorded at a $1 \mathrm{hr}$ nerve-muscle contact. This was a stable contact whose characteristics did not show much variation with time during the 30 min recording period. In all, 587 evoked events and 199 spontaneous events were obtained. A series of QSs were tested by MMRV. As shown in Figure 11, the procedure yielded optimal fit using Poisson statistics and a quantal size of $94 \mathrm{pA}$. The calculated mean quantal content was 2.44. The MRV also showed a sharp minimum. Thus, there was a choice of single quantal size which resulted in an excellent fit between the theoretical Poisson values and the quantized histogram of the actual data. Figure 11 also demonstrates that slight variations in the choice of the QS produced Poisson distributions quite different from the observed data. The other statistics produced poor fit in general. The only exception was binomial statistics at low $p$ and high $N$, which is equivalent to Poisson statistics. It may be noted that, in principle, MMRV procedure should be performed using smallest possible bin size. We have indeed started the procedure from a bin size of $2 \mathrm{pA}$, which is the size of current flow through a single $\mathrm{ACh}$ receptor channel. It yielded the same QS as that obtained by using the bin size $31 \mathrm{pA}$ (Fig. 11). The latter case was used for illustration.

This analysis predicted a QS that does not coincide with the peak of the amplitude histogram of the spontaneous events (Fig. $10 \mathrm{~A}$, inset). Similar results were found for 2 other sets of data of evoked responses. Perhaps there is a unique pool of quanta releasable only upon depolarization. Alternatively, a subpopulation of the spontaneous events could be the building blocks for the evoked response. The method of MMRV yields a value 

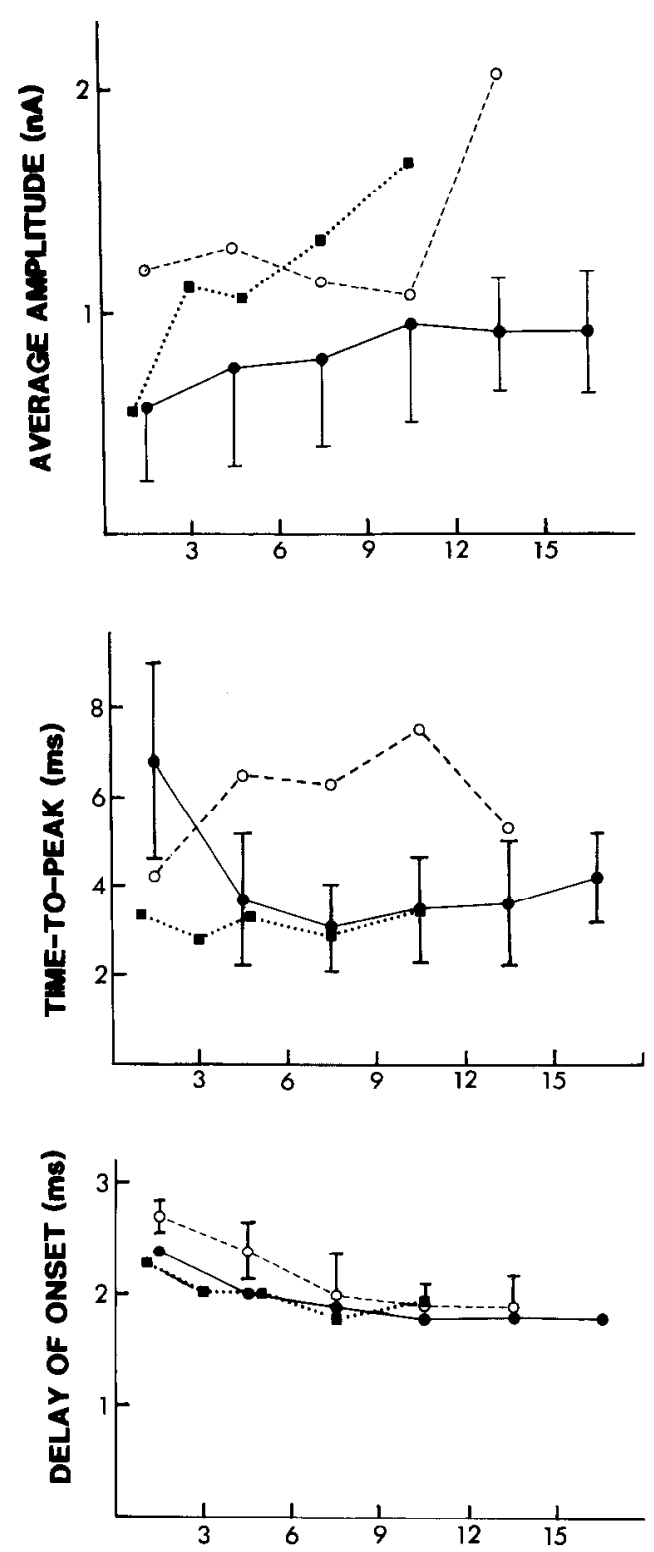

TIME AFTER CONTACT (min)

Figure 9. Postcontact changes in evoked synaptic currents. Data for the delay of onset (from the initiation of stimulus at the soma to the onset of evoked current at the muscle), the time-to-peak, and the amplitude of the evoked synaptic currents recorded from 3 pairs of manipulated neurite-myoball contacts were plotted. Different symbols refer to different contacts. Data represented by circles ( $\bullet$ were from the contact described in Figure 8. For clarity, only samples of error bars (SD) were shown. The increase in the average amplitude and the decrease in the delay of onset are statistically significant ( $t$ test, $p<0.05$ ) when the first and the last data points were compared.

for the mean QS while providing no information about variations in that size. If the variance of the quantal unit distribution were also known, then one could apply the original method of del Castillo and Katz (1954) to generate a theoretical evoked response curve with peaks and valleys that should closely follow the actual evoked data. In the absence of a known variance, a set of arbitrary variances was chosen, and theoretical evoked response curves were constructed. The variance that yielded the best fit ( $\mathrm{SD}$ of $31 \mathrm{pA}$ ) is used to generate the theoretical curve, which is compared with the actual data in Figure $10 B$. The general alignment of peaks and valleys gives further support that the MMRV method has yielded the correct quantal size.

\section{Effects of depleting extracellular $\mathrm{Ca}^{2+}$}

Influx of $\mathrm{Ca}^{2+}$ is required for triggering stimulus-evoked transmitter release at the mature neuromuscular junction (Katz and Miledi, 1967). We have examined whether a similar requirement exists for transmitter release at the early neurite-muscle contacts. $\mathrm{Ca}^{2+}$ was removed from the contact region by local perfusion with $\mathrm{Ca}^{2+}$-free saline (Ringer's solution with no $\mathrm{Ca}^{2+}$ ) through a micropipet. Figure 12 shows an example of the recording from a $1 \mathrm{hr}$ contact. Prior to the perfusion, both spontaneous and evoked synaptic currents were recorded from the muscle. The suprathreshold stimuli for the evoked release were applied through an extracellular micropipet to the soma of the neuron at a rate of $6 / \mathrm{min}$. Perfusion with $\mathrm{Ca}^{2+}$-free saline quickly abolished the evoked synaptic currents, which gradually recovered after the perfusion was terminated. In this example, the frequency of spontaneous synaptic currents was reduced during the perfusion. In other cases (see Fig. 13), different degrees of reduction was observed. In total, we have tested 13 neuritemyocyte pairs. Under the condition of $\mathrm{Ca}^{2+}$-free perfusion that completely abolished the evoked currents, the frequency and the mean amplitude of the spontaneous currents were unchanged in 5 cases; either or both were reduced in 6 cases; frequency was increased in the remaining 2 cases. We also noted that in 7 out of 13 cascs, SSC frequency was elevated following the return to normal $\mathrm{Ca}^{2+}$ concentration, as compared with that before the perfusion.

The reduction of SSC frequency during the perfusion could be interpreted in 2 different ways: There may exist 2 populations of SSCs, only one of which is dependent on the external $\mathrm{Ca}^{2+}$ concentration; alternatively, extracellular $\mathrm{Ca}^{2+}$ depletion may lead to a general reduction in the amount of $\mathrm{ACh}$ release per pulsatile event, so the lower frequency of events may have been due to reduction of the amplitude of some of the SSCs to an undetectable level. The reason for the increase of SSC frequency after the perfusion in many cases was unknown. It is clear, however, that the mechanism responsible for regulating evoked $\mathrm{ACh}$ release is substantially different from that for spontaneous release.

\section{Discussion}

\section{Studying early events by cell manipulation}

By studying synaptogenesis in cell culture systems, the early interaction between growing neurites and target muscle cells is now beginning to be understood. In order to study the early events, we controlled the timing of cell-cell contact by manipulating spherical myocytes (myoballs) into contact with co-cultured neurons. Myoballs in our Xenopus cultures arise as a result of using a clean, uncoated glass surface as the culture substratum. They are less adherent to the surface than spindle- or flat-shaped myocytes in the same culture and are thus more amenable to cell manipulation. We have found no evidence that the myoballs belong to a separate subpopulation of myocytes, with physiological properties different from those of the spindle and flat myocytes. The present study also shows that the characteristics of early spontaneous synaptic currents following the manipulated contact are nearly identical to those of the natural contact between the neurite and the spindle myocyte. Evoked synaptic currents at the spindle and flat myocytes were also similar to 

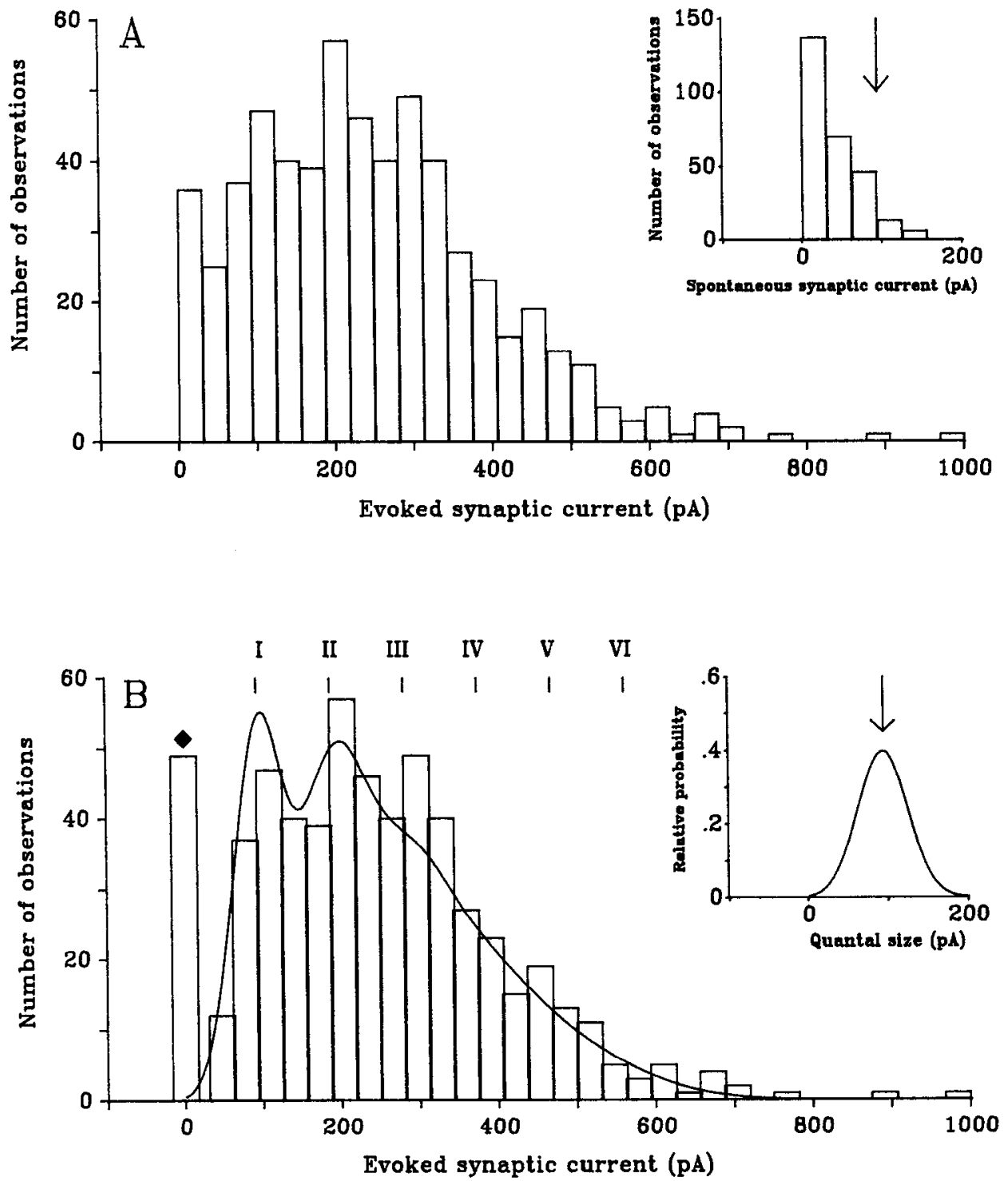

Figure 10. Quantal analysis of evoked synaptic currents. $A$, Amplitude histogram of evoked synaptic currents at a $1 \mathrm{hr}$ contact, constructed with a bin width of $31 \mathrm{pA}$. There were 587 trials. The mean response was 229 pA. Inset, Amplitude histogram of 199 spontaneous events. Note that the latter are not Gaussian-distributed around a mean, which prevented the use of conventional methods for quantal analysis. $B$, Inset depicts a chosen unit quantal distribution, with a SD of $31 \mathrm{pA}$ (1 bin) and a mean equal to that predicted by MMRV, i.e., 94 pA (3 bins). The method of Boyd and Martin (1956) was then used to generate a theoretical evoked response curve, based on Poisson statistics. Note the alignment of peaks and valleys between the curve and actual observed histogram. The evoked histogram is slightly modified from that shown in $A$ because all events less than half of the unit quantal size ( 1.5 bin in present case) are failures by definition. The diamond $(\$)$ shows the predicted number of failures. The Roman $n u$ merals refer to the amplitudes corresponding to multiples of chosen quantal unit. The arrows in $A$ and $B$ are at the best-fit quantal size predicted by the MMRV method. those described in this report (D. Sanes and M.-M. Poo, unpublished observations). In addition, ultrastructural studies (Buchanan et al., 1989) indicate that the myoball undergoes similar myofibrillar differentiation as the spindle myocyte and shows the same morphological changes following neurite contact.

\section{Pulsatile and quantal $A C h$ release}

Previous work (Xie and Poo, 1986) has shown that one of the first events following nerve-muscle contact is the induction of pulsatile release of $\mathrm{ACh}$ from the neuron. This induction depends solely on the physical contact between the 2 membranes, not any diffusible factor(s) released by the muscle cell, since a piece of excised muscle membrane is equally effective for induction. The ACh release induced by the contact results in spontaneous synaptic currents of markedly different amplitudes in the muscle cell. In the present study we have analyzed the origin of this amplitude variation in more detail. The result suggests that the most likely cause is variation in the amount of $\mathrm{ACh}$ released in each pulse event and/or in the extent of close membrane apposition surrounding various release sites. A larger area of close apposition may lead to more effective impediment of
ACh diffusion from the contact zone, hence larger amplitude of synaptic currents. However, it is not clear what could cause the difference in membrane apposition at various release sites or whether such apposition can form within seconds following the contact, leading to the rapid appearance of SSCs of markedly different amplitudes (see Fig. 2C).

If the amount of $\mathrm{ACh}$ in each pulse of spontaneous release varies, it may vary continuously or in a quantal fashion. In the latter case, there is a fixed unit for spontaneous ACh release and large spontaneous currents are due to simultaneous release of several units. The possible existence of a quantal unit for the spontaneous current has not yet been studied in this preparation.

The evoked release during the early phase of contact shows substantial amplitude fluctuation. Statistical analysis of the synaptic currents indicate that observed evoked response is consistent with the quantal hypothesis of transmitter release, and that the quantal unit underlying the evoked response corresponds to only a small subpopulation of the observed spontaneous events. Two populations of spontaneous synaptic potentials have also been identified by previous studies on developing nerve-muscle synapses in similar Xenopus cultures (Kidokoro, 


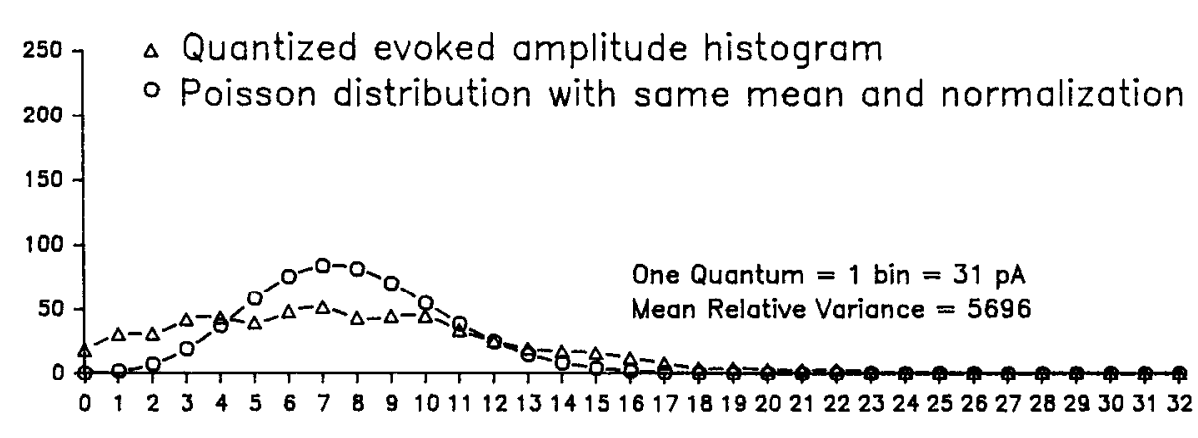

Figure 11. Identification of quantal size (QS) by minimization of mean relative variance (MMRV). The figure shows the results of a series of theoretical computations to find the MMRV. Single QS from 1 bin (31 pA, top) to 5 bins $(156 \mathrm{pA}$, bottom) were used. Each graph compares the "quantized" evoked histogram $(\triangle)$, constructed from the original observed histogram (Fig. 10A), with the theoretical Poisson distribution with the same mean and normalization (O). Only for the choice of 1 quantum equals 3 bins, or at minimal MRV, do the 2 histograms line up. Note that equal distances along the $x$ axis correspond to equal current amplitudes.
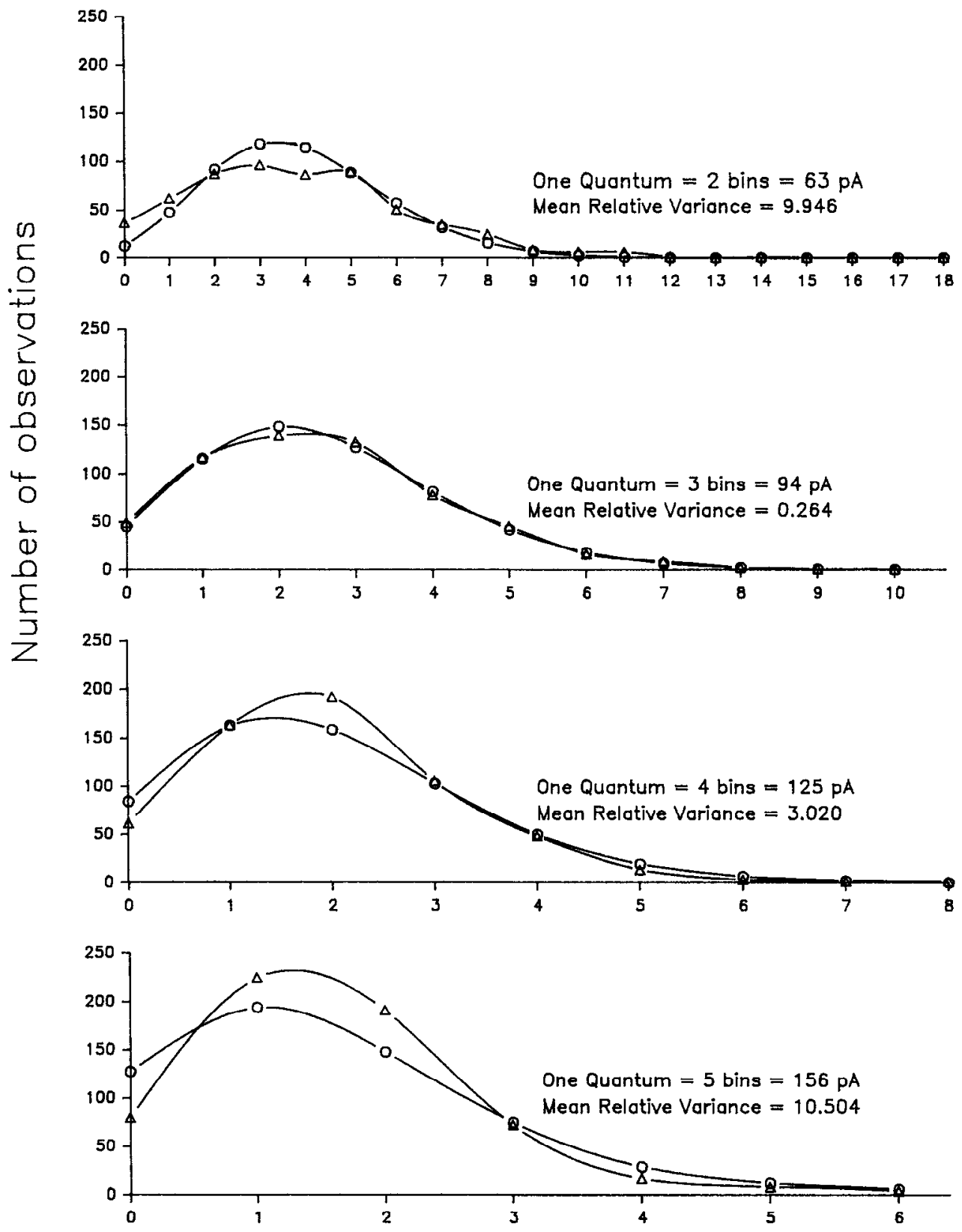

\section{Number of quanta released}

1984). At the mature synapse there is only one population of spontaneous events, which corresponds to the quantal unit of the evoked response. Whether such a developmental change of spontaneous ACh release plays an important function in the maturation of the synapse or simply reflects structural changes in the nerve terminal remains to be studied.

Regardless of whether the quantal unit exists for either spontaneous or evoked events, the pulsatile nature of the sponta- 


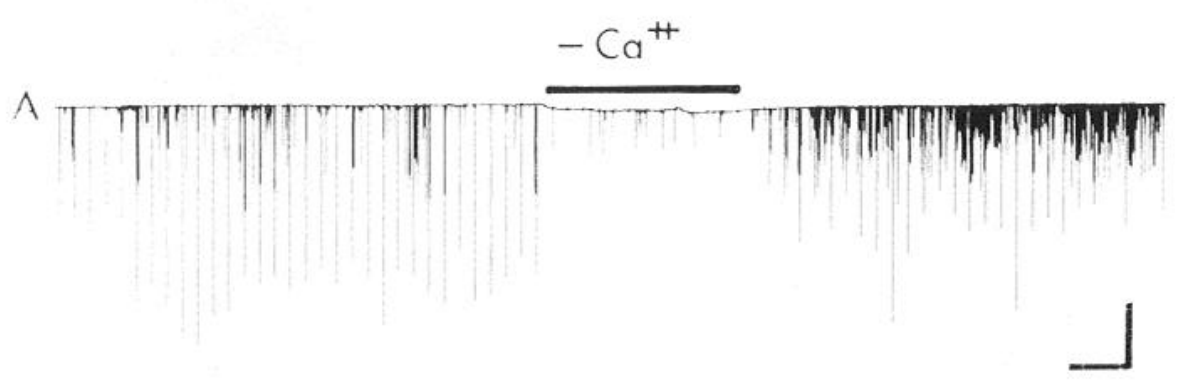

B

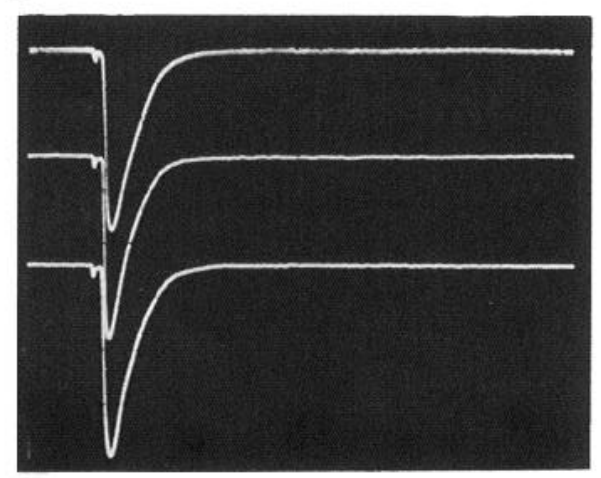

C

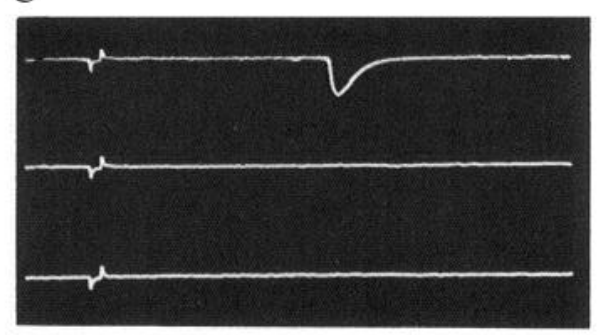

D

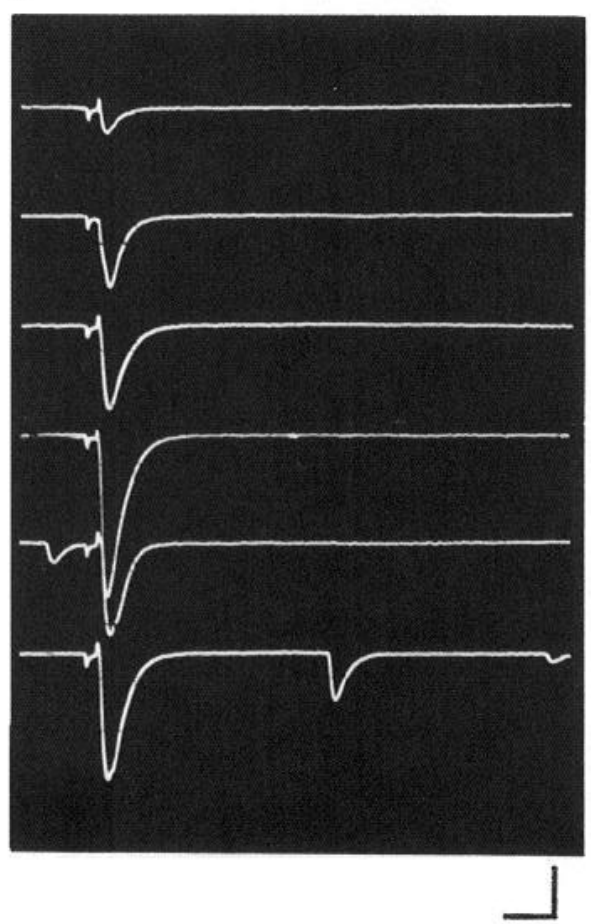

Figure 12. Effects of the depletion of extracellular $\mathrm{Ca}^{2+}$. Ringer's solution containing no $\mathrm{Ca}^{2+}$ was locally applied to a neurite-myoball pair that had been in contact for a period of about $1 \mathrm{hr}$. The neuron was stimulated at the soma at a rate of $6 / \mathrm{min}$. $A$, The membrane currents recorded at the myoball under voltage-clamp conditions $\left(V_{m}=-70\right.$ $\mathrm{mV})$. The duration of $0 \mathrm{Ca}^{2+}$ perfusion is marked by the bar above the trace. Scales: $40 \mathrm{sec}$ and 500 pA. $B-D$, Samples of synaptic currents before, during, and after the perfusion, respectively, shown in higher time resolution. Note the presence of a spontaneous event and the complete absence of evoked response during the period of $0 \mathrm{Ca}^{2+}$ perfusion in $C$. Scales: $10 \mathrm{msec}, 500 \mathrm{pA}$. neous release is consistent with the notion that vesicular exocytosis is the basis for transmitter release. Clear vesicles similar in appearance to synaptic vesicles were found in the cytoplasm of the Xenopus spinal neurons that showed spontaneous and evoked ACh release, although well-defined active zone structures were not observed during the first few hours of nervemuscle contact (Buchanan et al.. 1989).

\section{Postcontact changes in synaptic currents}

During the first $20 \mathrm{~min}$ following the nerve-muscle contact, there were significant changes in the characteristics of both the SSCs and ESCs. Some of these changes, namely, the increase in the mean amplitude of both currents, the increase in the frequency of SSCs, and the decrease in the delay of onset of the ESCs, may be attributed to an increase in the total area and/or the closeness of apposing nerve and muscle surfaces. The adhesion measurement showed that high level nerve-muscle adhesion also develops during the first 15 min of contact. Adhesion between the nerve and muscle cells reflects binding of surfacebound molecules on 2 opposing surfaces. This binding may lead to a "zippering" effect that increases the contact area and reduces the distance between the membranes. Ultrastructural examination of the early nerve-muscle contacts in this culture $(\mathrm{Bu}-$ chanan et al., 1989) has suggested such zippering of membranes during the early phase of the contact. Since the induction of $A C h$ release depends upon the physical contact with the muscle membrane, a larger area of contact with neurite should result in a higher frequency of SSCs through higher levels of induction. In addition, increased membrane apposition helps to bring receptive muscle surface to additional release sites that were previously beyond the receptive range of the muscle membrane. Close membrane-membrane apposition should also reduce the rise time and increase the amplitude of the synaptic currents. We did not observe statistically significant reduction of rise times of SSCs and ESCs in most cases. This may due to the fact that the amount of reduction and the number of current samples examined were too small.

The notion that the change in membrane-membrane apposition accounts for the changes in synaptic currents was also supported by the results from the repeated contact experiments. We found that increasing pressure on the myoball-neurite contact, which presumably increases the total area of close membrane apposition, led to an immediate increase in the frequency and mean amplitude and a reduction of mean rise time of the SSCs. We also noted that the variability in the postcontact changes between different pairs of the manipulated contacts (see 
TIME (min)

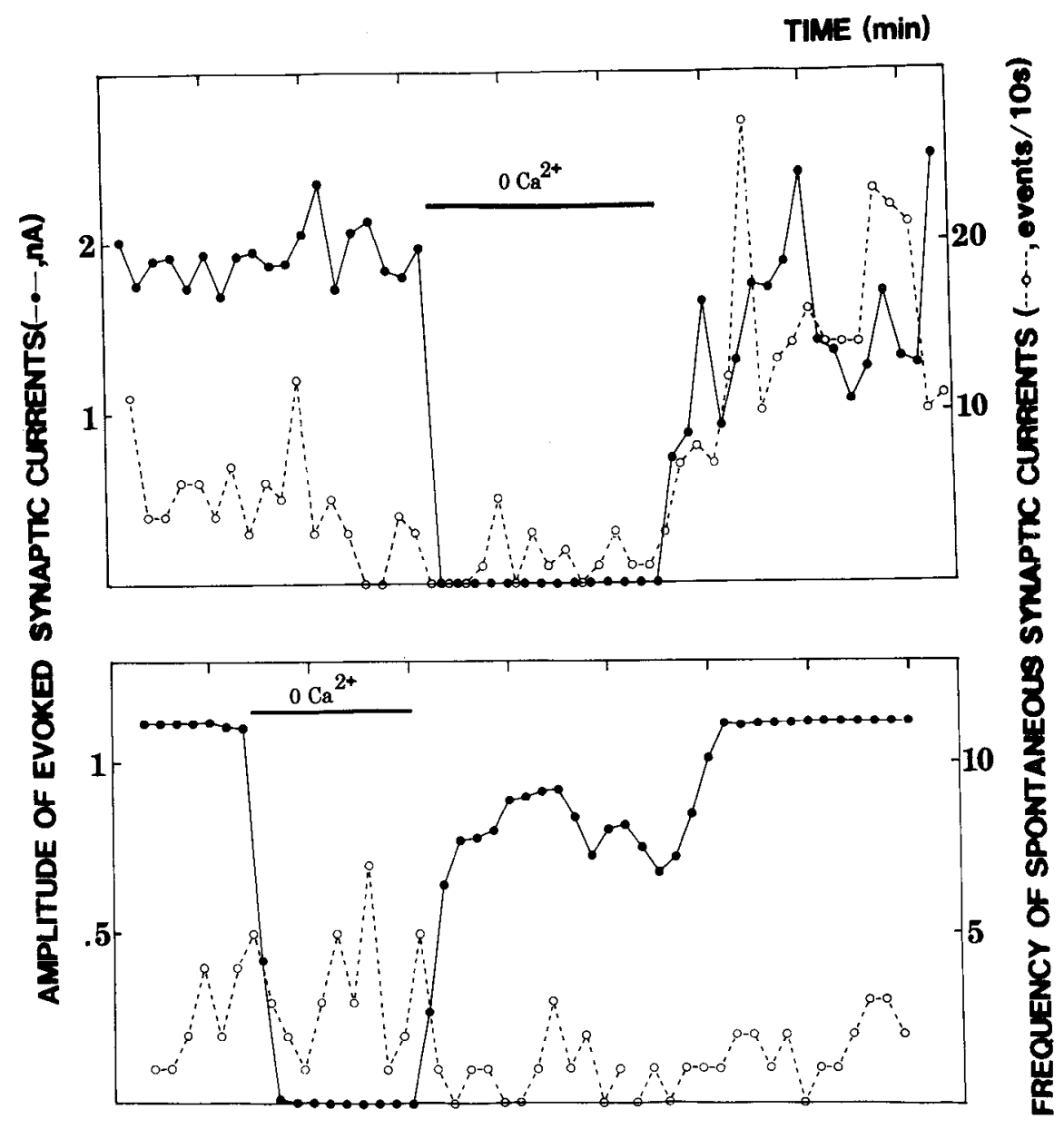

Figure 13. Analysis of the effects of $\mathrm{Ca}^{2+}$ depletion. The amplitude of evoked synaptic currents and the frequency of spontaneous synaptic currents were plotted for 2 separate experiments, including that described in Figure 12. The periods of $0 \mathrm{Ca}^{2+}$ perfusion were marked by the complete absence of the evoked response. The frequency of the spontaneous events was either slightly reduced (upper diagram) or apparently unaffected (lower diagram). In the former case, the frequency showed a striking increase following the return of normal saline.

Table 1) is likely to result from different degrees of initial membrane-membrane apposition produced directly by manipulation.

Postcontact increase in the total area of membrane-membrane apposition can lead to a higher amplitude of synaptic currents by bringing more $\mathrm{ACh}$ receptors within the effective range of released $\mathrm{ACh}$, without any change in the receptor density on the muscle surface. However, studies of $\mathrm{ACh}$ receptor topography on the muscle surface before and after innervation by co-cultured neurons (Anderson et al., 1977; Frank and Fischbach, 1979) have shown that neuritic processes can induce formation of ACh receptor clusters along the path of neurite-muscle contact. Kidokoro et al. (1980) observed changes in the properties of spontaneous synaptic potentials in Xenopus cell culture that correlate with the accumulation of $\mathrm{ACh}$ receptors at the nerve contact site: Muscle cells that showed synaptic potentials with higher frequencies, shorter rise times, and larger amplitudes also tended to have more extensive clustering of ACh receptors. In the latter study, the duration of neurite-muscle contact was uncertain. If the ACh clustering could occur in the order of $10 \mathrm{~min}$, then the postcontact changes in the synaptic currents observed in the present study could also be attributed to a local increase of ACh receptor density, in addition to the change in membrane-membrane apposition. Recent in vivo observation of ACh receptor clustering in Xenopus embryo (M. Cohen, personal communication) suggests that small clusters of $\mathrm{ACh}$ receptors could indeed occur on the order of minutes.

\section{Early transition of synaptic efficacy}

One of the most intriguing aspects of neuronal development is the specificity in the formation of neuronal connections. What are the properties that characterize a specific synapse? A functional synapse can form without much specificity: Growth cones can secret transmitter without contact with the target cell (Hume et al., 1983; Young and Poo, 1983; Sun and Poo, 1987), and any cell that contains the proper transmitter receptors will respond to a nearby secreting growth cone with an evoked response without any specific contact-mediated surface recognition of the neuron. It has been shown that functional synapses between cholinergic retinal neurons and skeletal muscles, which are inappropriate as partners in vivo, exists only a short period in culture (Puro et al., 1977). Therefore, one must look for temporal changes in the structural and functional properties of the synapse, other than the mere initial occurrence of nerveevoked synaptic response, to ascertain the establishment of a specific and appropriate connection.

A possible indication of specific synapse formation is the postcontact transition of synaptic efficacy. As discussed above, such a transition is likely to depend upon morphological changes associated with the formation of extensive close membrane apposition between the pre- and postsynaptic cells. The close membrane apposition, or the "7ippering" of membranes (see Buchanan et al., 1989) may reflect the formation of molecular bonds between the contacting surfaces, a process inherent in the 
contact-mediated cell-cell recognition. A recent model study of cell-cell recognition and adhesion (McCloskey and Poo, 1986) suggests that if the recognition event involves binding of recognition molecules bound to the opposing surfaces, then the recognition process will result in an accumulation of these molecules at the contact zone-a natural consequence of the lateral mobility of membrane-bound molecules. Furthermore, the morphological consequence of such molecular localization is the zippering of contacting membranes and the formation of extensive close membrane apposition. The latter study also suggests that the accumulation of recognition/adhesion molecules at the contact site is responsible for the post contact gradual stabilization of adhesion, since both of the latter 2 processes require a time in the order of $10 \mathrm{~min}$ to complete. Increased synaptic efficacy with time after contact has also been observed previously at synapses between cultured chick ciliary ganglion neurons and myotubes (Role et al., 1987).

Taken together, our observations of the transition of synaptic efficacy and the development of nerve-muscle adhesion could be nicely integrated by the hypothesis that the postcontact increase in synaptic efficacy results from the zippering of nervemuscle membranes, which in turn reflects the bonding and localization of specific nerve and muscle surface recognition/adhesion molecules at the site of contact. Such transition during the early postcontact period could thus signify specific synaptogenesis. The identity of these recognition/adhesion molecules on the nerve and muscle surface, if they exist at all, remains to be elucidated.

\section{Conclusion}

The studies of nerve-muscle interaction during the early stages of synaptogenesis reveal the rapidity and complexity of the structural and functional changes that occur during development. The analysis of the synaptic currents and the cellular basis for the changes in their characteristics provides a useful approach to the study of the structure-function relationship in synaptic physiology. The transition from low- to high-efficacy synaptic transmission and the concurrent increase in nervemuscle adhesion suggest that early transition of synaptic efficacy may signify establishment of specific synaptic connection.

\section{References}

Anderson, M. J., M. W. Cohen, and E. Zorychta (1977) Effects of innervation on the distribution of acetylcholine receptors on cultured muscle cells. J. Physiol. (Lond.) 268: 731-756.

Boyd, I. A., and A. R. Martin (1956) The end-plate potential in mammalian muscle. J. Physiol. (Lond.) 132: 74-91.

Buchanan, J., Y.-a. Sun, and M.-m. Poo (1989) Studies of nervemuscle interactions in Xenopus cell culture: Fine structure of early functional contacts. J. Neurosci. 9: 1540-1554.

Chao, N.-M., S. H. Young, and M.-M. Poo (1981) Localization of cell membrane components by surface diffusion into a "trap." Biophys. J. 36: 139-153.

Chow, I., and M.-M. Poo (1985) Release of acetylcholine from embryonic neurons upon contact with the muscle cell. J. Neurosci. 5: 1076-1082.

del Castillo, J., and B. Katz (1954) Quantal components of the endplate potential. J. Physiol. (Lond.) 124: 560-573.

Frank, E., and G. D. Fischbach (1979) Early events in neuromuscular junction formation in vitro. Induction of acetylcholine receptor clusters in the postsynaptic membrane and morphology of newly formed nerve-muscle synapses. J. Ccll Biol. 83: 143-158.

Hamill, O. P., A. Marty, A. Neher, B. Sakmann, and F. J. Sigworth (1981) Improved patch-clamp techniques for high-resolution current recording from cells and cell-free membrane patches. Pfluegers Arch. 391: $85-100$.

Hume, R. I., L. W. Role, and G. D. Fischbach (1983) Acetylcholine release from growth cones detected with patches of acetylcholine receptor-rich membranes. Nature 305: 632-634.

Katz, B., and R. Miledi (1967) The timing of calcium action during neuromuscular transmission. J. Physiol. (Lond.) 189: 535-544.

Kidokoro, Y. (1984) Two types of miniature endplate potentials in Xenopus nerve-muscle cultures. Neurosci. Res. 1: 157-170.

Kidokoro, Y., and E. Yeh (1982) Initial synaptic transmission at the growth cone in Xenopus nerve-muscle cultures. Proc. Natl. Acad. Sci. USA 79: 6727-6731.

Kidokoro, Y., M. J. Anderson, and R. Gruener (1980) Changes in synaptic potential properties during acetylcholine receptor accumulation and neurospecific interaction in Xenopus nerve-muscle cell culture. Dev. Biol. 78: 464-483.

Martin, A. R. (1977) Junctional transmission: Presynaptic mechanism. In Handbook of Physiology, S. R. Geiger, ed., pp. 329-355, American Physiological Society, Baltimore, MD.

McCloskey, M., and M.-M. Poo (1986) Contact-induced redistribution of specific membrane components: Local accumulation and development of adhesion. J. Cell Biol. 102: 2185-2196.

Moody-Corbett, F., P. R. Weldon, and M. W. Cohen (1982) Cholinesterase localization at sites of nerve contact on embryonic amphibian muscle cells in culture. J. Neurocytol. 11: 381-394.

Nieuwkoop, P. D., and J. Faber (1967) Normal Table of Xenopus laevis, 2nd ed., North Holland, Amsterdam.

Poo, M.-M. (1982) Rapid lateral diffusion of functional ACh receptors in embryonic muscle membrane. Nature 295: 332-334

Puro, D. G., F. G. DeMello, and M. Nirenberg (1977) Synapse turnover: The formation and termination of transient synapses. Proc. Natl. Acad. Sci. USA 74: 4977-4981.

Role, L. W., D. G. Roufa, and G. D. Fischbach (1987) The distribution of acetylcholine receptor clusters and sites of transmitter release along chick ciliary ganglion neurite-myotube contacts in culture. J. Cell Biol. 104: 371-379.

Spitzer, N. C., and J. C. Lamborghini (1976) The development of the action potential mechanism of amphibian neurons isolated in culture. Proc. Natl. Acad. Sci. USA 73: 1641-1645.

Sun, Y.-A., and M.-M. Poo (1987) Evoked release of acetylcholine from growing embryonic neuron. Proc. Natl. Acad. Sci. USA 84: 2540-2544.

Xie, Z.-P., and M.-M. Poo (1986) Initial events in the formation of neuromuscular synapse: Rapid induction of acetylcholine release. Proc. Natl. Acad. Sci. USA 83: 7069-7073.

Young, S., and M.-M. Poo (1983) Spontaneous release of transmitter from growth cone of embryonic neuron. Nature 305: 634-637. 